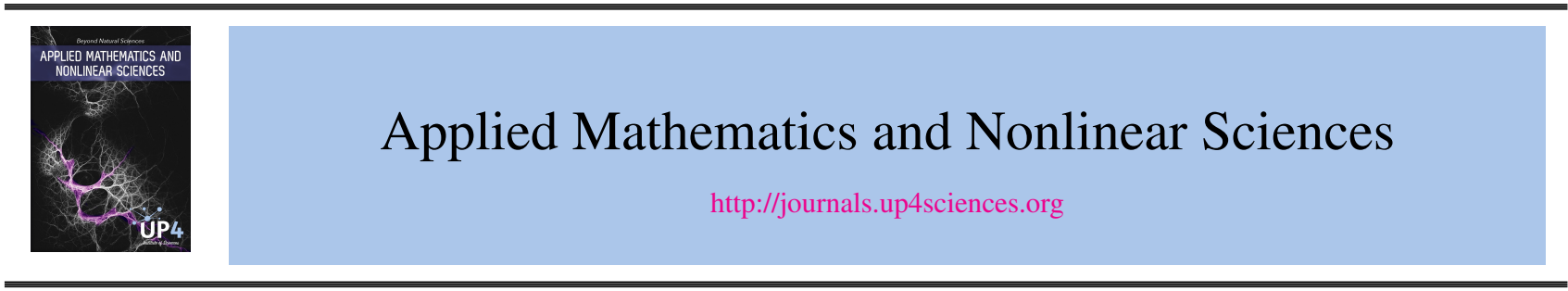

\title{
Non-autonomous perturbations of a non-classical non-autonomous parabolic equa- tion with subcritical nonlinearity
}

\author{
Matheus C. Bortolan ${ }^{1 \dagger}$, and Felipe Rivero ${ }^{2 \ddagger}$. \\ ${ }^{1}$ Departamento de Matemática, Centro de Ciências Físicas e Matemáticas, UFSC \\ BRAZIL \\ ${ }^{2}$ Departamento de Análise, Instituto de Matemática e Estatística, UFF \\ BRAZIL
}

Submission Info

Communicated by Tomás Caraballo

Received 10th October 2016

Accepted 8th February 2017

Available online 8th February 2017

\begin{abstract}
In this work we study the continuity of four different notions of asymptotic behavior for a family of non-autonomous non-classical parabolic equations given by

$$
\left\{\begin{array}{l}
u_{t}-\gamma(t) \Delta u_{t}-\Delta u=g_{\varepsilon}(t, u), \text { in } \Omega \\
u=0, \text { on } \partial \Omega .
\end{array}\right.
$$

in a smooth bounded domain $\Omega \subset \mathbb{R}^{n}, n \geqslant 3$, where the terms $g_{\varepsilon}$ are a small perturbation, in some sense, of a function $f$ that depends only on $u$.
\end{abstract}

Keywords: Non-autonomous perturbations; non-autonomous dynamical systems; pullback attractors; cocyle attractor; uniform attractor; non-classical parabolic equations; evolution process; skew-product semiflow

AMS 2010 codes: 37B55, 35K55, 34D45, 35Q74, 35B20

†Corresponding author.

Email address: m.bortolan@ufsc.br

†Email address: lf_rivero@id.uff.br 


\section{Introduction}

\subsection{Asymptotic behavior of non-autonomous equations}

What is the difference between the asymptotic behavior of an autonomous and a non-autonomous equation? This may be, at first glance, a simple question to answer. Let us discuss this problem a little.

Consider a general non-autonomous differential equation given by

$$
\left\{\begin{array}{l}
\dot{u}=F(t, u), t \geqslant s, \\
u(s)=u_{0} \in X,
\end{array}\right.
$$

where $X$ is a Banach space and $F$ is in some metric space $\mathfrak{C}$ of functions. Assume also that for each $u_{0} \in X$, $s \in \mathbb{R}$ and $F \in \mathfrak{C}$, the problem (1) has a uniquely defined solution $u\left(t, s, F, u_{0}\right)$ for all times $t \geqslant s$ and the map $\left(t, s, u_{0}\right) \mapsto u\left(t, s, F, u_{0}\right)$ is continuous for each $F \in \mathfrak{C}$.

We know that, when $F$ is independent of time, $u\left(t, s, F, u_{0}\right)=u\left(t-s, 0, F, u_{0}\right)$, that is, the dependence of $t$ and $s$ in $u$ are artificial, and in fact $u$ depends only on the elapsed time $t-s$. Hence, the asymptotic behavior (the behavior for large times) can be obtained making $t \rightarrow \infty$ or $s \rightarrow-\infty$, indistinctly.

However, if $F$ is time dependent, the dependence of $t$ and $s$ of the solution is explicit and the scenarios arising from making $t \rightarrow \infty$ and $s \rightarrow-\infty$ may be completely different. This is not so surprising once we realize that in the autonomous case ( $F$ independent of time) we have only one vector field, namely $F$, driving the solutions but, in the non-autonomous case we have infinitely many vector fields $(F(t, \cdot)$ for each $t)$ driving the solutions, and their behavior may be completely different for $t \rightarrow \infty$ and $s \rightarrow-\infty$. This clarifies a little the understanding of asymptotic behavior for non-autonomous equations, and shows that it is not an easy task to study this subject. First, one must define which behavior will be treated: the forward attraction (when $t \rightarrow \infty$ ) or the pullback attraction (when $s \rightarrow-\infty$ ). Once the framework is set, we reach another problem: in any of them it is clear that the asymptotic behavior of the solutions of (1) are related with the behavior of the vector fields $F(t, \cdot)$ when $t \rightarrow \pm \infty$, which can be unrelated with the behavior of each $F(t, \cdot)$. This could have been said in another way: the translates $\theta_{s} F$ alone are not enough, in general, to describe the asymptotic dynamics of (1) (for more details on this subject we refer to [7] or $[9,16,17])$. Thus, the next question comes quite naturally: how do we introduce the limiting vector fields of $F(t, \cdot)$ in the study?

Consider $\mathfrak{C}$ the space of all functions $H: \mathbb{R} \times X \rightarrow X$, that are bounded in sets of the form $\mathbb{R} \times B$, where $B$ is a bounded set of $X$. Consider also the shift operator $\theta_{t}: \mathfrak{C} \rightarrow \mathfrak{C}$ given by

$$
\theta_{t} H(\cdot, \cdot)=H(t+\cdot, \cdot), \text { for each } t \in \mathbb{R} .
$$

Now define $\Sigma_{0}=\left\{\theta_{t} F\right\}_{t \in \mathbb{R}}$, which is the set of all translations of $F$ and let

$$
\Sigma=\text { closure of } \Sigma_{0} \text { in } \mathfrak{C},
$$

which is known as the hull of $F$.

Using our assumptions for the problem (1), we know that each problem

$$
\left\{\begin{array}{l}
\dot{u}=H(t, u), \text { for } t \in \mathbb{R} \\
u(0)=u_{0} \in X,
\end{array}\right.
$$

has a uniquely defined solution $\varphi(t, H) u_{0}$ for each $t \geqslant 0, u_{0} \in X$ and $H \in \Sigma$.

Note that we are now dealing with all the solutions of the problem (1) but also with all the solutions of the limiting vector fields of $F(t, \cdot)$.

Remark 1. To obtain problem (1), just consider $H=\theta_{S} F$. The solutions $\xi$ of (1) and $\psi$ of (2) in this case are related by

$$
\psi(\cdot)=\xi(\cdot+s) .
$$


These objects, namely the solutions $\varphi(t, H) u_{0}$ in $X$ and the shift operator $\theta_{t}$ in $\Sigma$ a, give rise to what we call a non-autonomous dynamical system ${ }^{\mathrm{b}}$. Several authors have studied this object, in the pursuit of fully understanding of the asymptotic dynamics of equation (1), and there are two distinct branches: the pullback approach (see, for example [16,22]), which deals with pullback attraction, and the uniform approacch (see, for example [28]). Each group has achieved several interesting results concerning asymptotic behavior of nonautonomous equations, and up until recently, these approaches seemed unrelated. In [5,7] the authors unify these results, presenting relations between these frameworks. To this end and to reach the full extent of this theory, they transform the non-autonomous dynamical system defined by $\varphi$ and $\theta_{t}$ in an autonomous one, non-trivially, by defining

$$
\Pi(t)\left(u_{0}, H\right)=\left(\varphi(t, H) u_{0}, \theta_{t} H\right),
$$

which is called the skew-product semiflow, and study the autonomous semiflow defined by $\Pi$ to obtain results for $\varphi$ simply analysing the canonical projection in the first coordinate.

To be a little more precise, inside the study of non-autonomous equations such as (1) we can distinct at least four different notions of attractors, namely:

(i) the global attractor for the skew-product semiflow;

(ii) the pullback attractor for the evolution process.

(iii) the cocyle attractor for the non-autonomous dynamical system and

(iv) the uniform attractor for the non-autonomous system.

We will give a detailed description of each one of these objects in Section 2, as well as the relationships between these concepts, as done in [7], to describe the non-autonomous problems (1) in a very complete way.

\subsection{Small perturbations}

Imagine now that we have not only a single $F(t, \cdot)$ but a family $\left\{H_{\varepsilon}(t, \cdot)\right\}_{\varepsilon \in[0,1]}$ such that $H_{\varepsilon}$ is close (in some sense) to $F$ as $\varepsilon \rightarrow 0$. Are we able to obtain results on the asymptotic behavior of the problems

$$
\left\{\begin{array}{l}
\dot{u}=H_{\varepsilon}(t, u), \text { for } t>0 \\
u(s)=u_{0} \in X
\end{array}\right.
$$

for $\varepsilon$ sufficiently small, given that we know the behavior of (1)?

Note that to study each problem one must perform all the previous discussion; that is, each $\varepsilon$ will generate a different non-autonomous dynamical system and a skew-product semiflow. The question is: can we obtain results of continuity of the different types of asymptotic behavior as $\varepsilon \rightarrow 0$ ?

This question, theoretical as is sounds, has a meaning in applications. Models in the real world are always approximations, due to data collection, empirical laws and simplifications, and thus, it is crucial that we are able to transfer properties from an equation to some small perturbations. Without this property, we have no guarantee whatsoever that the real phenomena will have a behavior close to our model.

In $[5,7]$, the authors provide and extensive study on this topic, giving a detailed study of non-autonomous dynamical systems, different scenarios of asymptotic behavior and relationships among them, extracting informations from the skew-product semiflow and transporting them to the non-autonomous dynamical system. Also, the reader can find a deep study of continuity of small perturbations of non-autonomous system, but arising from autonomous equations (see for instance [7,15, 16]).

The study of non-autonomous perturbations of non-autonomous dynamical systems directly is still an almost blank page, and in this paper we give some steps in this direction, by studying non-autonomous perturbations

\footnotetext{
${ }^{a}$ Clearly we can consider the restriction of the shift operator $\theta_{t}$ to $\Sigma$.

${ }^{\mathrm{b}}$ See Definition 5.
} 
of a non-autonomous equation, to provide results of continuity of the asymptotic behavior using the framework discussed above.

It is not known, so far, how to do the general theory when we consider non-autonomous perturbations of a non-autonomous system. In examples, we can see however that few steps are clear: first one must be able to prove the global existence and uniqueness of solutions not only for the equation in question, but also for all the limiting vector fields associated with the non-linearity - this step is the key for the development of the following results - to be able to construct a non-autonomous system. Then we must be able, with some uniformity on the vector fields, to obtain an uniform estimate that allow us to find a compact set that attracts all the solutions, independently of the vector field. Once this is done, we can find such an attractor for the associated skew-product semiflow and with this object at hand, we can try to understand all the asymptotic behaviors of our equation. Dealing with perturbations adds a difficulty to this process, since we must be able to do such study for each $\varepsilon$ small and obtain the result with uniformity in this parameter.

\subsection{Non-autonomous non-classical parabolic equations}

As mentioned before, a general theory for the study of non-autonomous perturbations of non-autonomous models is not available at this point, so we will put our best efforts to understand in some elaborated examples, in order to obtain a deep understanding of such perturbations. The problem we will deal with in this paper is to study non-autonomous perturbations of some non-autonomous parabolic equations.

Non-classical parabolic equations arise as models describing physical phenomena such as non-Newtonian flow, soil mechanics, heat conduction, etc. (see, for instance, $[1-4,8,21,23,25,29,30]$ and references therein). We will focus our study in non-autonomous perturbations of the following non-classical non-autonomous parabolic equation

$$
\left\{\begin{array}{l}
u_{t}-\gamma(t) \Delta u_{t}-\Delta u=f(u), \text { in } \Omega \\
u=0, \text { on } \partial \Omega
\end{array}\right.
$$

where $\Omega \subset \mathbb{R}^{n}$ is a smooth bounded domain, for some $n \geqslant 3$, with $f$ and $\gamma$ satisfying some suitable conditions. More specifically, we will deal with perturbations of the form

$$
\left\{\begin{array}{l}
u_{t}-\gamma(t) \Delta u_{t}-\Delta u=g_{\varepsilon}(t, u), \text { in } \Omega \\
u=0, \text { on } \partial \Omega
\end{array}\right.
$$

where $\left\{g_{\varepsilon}\right\}_{\varepsilon \in[0,1]}$ is a family of non-autonomous functions satisfying some continuity conditions.

In the work of Aifantis et al., [1-3] we can find a quite general approach to deduce these equations in the autonomous case without delay. In the aforementioned papers, it is pointed out that the classical reactiondiffusion equation

$$
u_{t}-\Delta u=g(u)
$$

does not contain each aspect of the reaction-diffusion problem, and it neglects viscidity, elasticity, and pressure of medium in the process of solid diffusion. The authors obtained a diffusion theory similar to Fick's classical model for solute in an undisturbed solid matrix, obtaining a hyperbolic equation

$$
u_{t}+D_{1} u_{t t}=D_{2} \Delta u
$$

where $D_{1}$ and $D_{2}$ are positive constants. Assign viscosity to the diffusing substance, they arrived to que following equation

$$
u_{t}+D_{1} u_{t t}=D_{2} \Delta u+D_{3} \Delta u_{t}
$$

and neglecting the inertia term, finally obtained the non-classical parabolic equation

$$
u_{t}=D_{2} \Delta u+D_{3} \Delta u_{t}
$$


where $D_{3}$ is also a positive constant.

The asymptotic behavior of the model without delay terms and with constant coefficients

$$
u_{t}-\mu \Delta u_{t}-\Delta u+g(u)=f(x), \quad \mu \in[0,1]
$$

is studied in [31], where, in particular, it is shown the well-posedness of the problem and the existence of the global attractor either in $H_{0}^{1}(\Omega)$ or in $H^{2}(\Omega)$, depending on the regularity of the initial data. They also showed the continuity of the global attractor in Hausdorff semidistance when $\mu \rightarrow 0$ in $H_{0}^{1}(\Omega)$.

The introduction of a time dependence in coefficient $\gamma(t)$ represents the variability of viscosity in time due to, for example, external environmental temperatures. This time dependence provides the system with a nonautonomous nature.

The study of a non-autonomous case with delay appeared in [12] for the first time, where it was established the well-posedness of the problem when $\gamma(t) \equiv \gamma$ is constant.

In [26], Rivero studied the existence of the pullback attractor and its continuity under non-autonomous perturbations, showing the existence of a concrete structure under some assumptions on the non-linearity and giving a first approach to the study of perturbations in non-autonomous problems.

Remark 2. This example is understood by us as a good starting point to the study of non-autonomous perturbations. Mainly because (2) is a non-autonomous equation, but term that causes this phenomena (the function $\gamma$ ) has no effect on the equilibria, which are the equilibria of the elliptic equation $-\Delta u=f(u)$.

\subsection{Novelties}

In this paper we give a step towards understanding non-autonomous perturbations of non-autonomous equations. We first study the problem (6) for each $\varepsilon \in[0,1]$ using the ideas presented in Subsection 1.1, but always having the discussion of Subsection 1.2 in mind, that is, not only we will deal with each equation separately for each $\varepsilon$, but also we have to take into account that we must be able to obtain the results with uniformity for $\varepsilon \in[0,1]$. Using this, we will be able to obtain continuity results for the family of equations given by (6). In the next section, we will present a detailed description of our main results.

Remark 3. One important thing to stress out is that, even that $f$ does not depend on $t$, the function $\gamma$ makes problem (5) non-autonomous.

\subsection{Description of the main results}

To describe the contents of our work and to state the main results, we first make some assumptions on the functions $\gamma, f$ and $g_{\varepsilon}$ as follows: suppose that $\gamma: \mathbb{R} \rightarrow(0, \infty)$ is a uniformly continuous function which satisfies $0<\gamma_{0} \leqslant \gamma(t) \leqslant \gamma_{1}<\infty$ and the family $\left\{g_{\varepsilon}\right\}_{\varepsilon \in[0,1]}$ of continuously differentiable functions from $\mathbb{R}^{2}$ to $\mathbb{R}$ with $g_{0}(t, s)=f(s)$ for all $t, s \in \mathbb{R}$, that satisfies

$$
\begin{gathered}
\left|g_{\varepsilon}\left(t, s_{1}\right)-g_{\varepsilon}\left(t, s_{2}\right)\right| \leqslant \alpha\left|s_{1}-s_{2}\right|\left(1+\left|s_{1}\right|^{\rho-1}+\left|s_{2}\right|^{\rho-1}\right), \\
\limsup _{|s| \rightarrow \infty} \frac{g_{\varepsilon}(t, s)}{s} \leqslant \delta<\lambda_{1}, \\
\int_{0}^{\infty} \frac{\partial}{\partial t} g_{\varepsilon}(t, s) d s<\infty
\end{gathered}
$$

Also we assume that there exists a bounded function $\beta$ defined in the interval $[0,1]$ with $\beta(\delta) \rightarrow 0$, as $\delta \rightarrow 0^{+}$, and satisfying

$$
\sup _{t \in \mathbb{R}}\left|g_{\varepsilon}(t, s)-f(s)\right| \leqslant \beta(\varepsilon)\left(1+|s|^{\rho-1}\right), \text { for all } s \in \mathbb{R} \text { and } \varepsilon \in[0,1],
$$


and

$$
\sup _{t \in \mathbb{R}}\left|\partial_{s} g_{\varepsilon}(t, s)-f^{\prime}(s)\right| \leqslant \beta(\varepsilon)\left(1+|s|^{\rho-1}\right), \text { for all } s \in \mathbb{R} \text { and } \varepsilon \in[0,1],
$$

where $\lambda_{1}>0$ is the first eigenvalue of the negative Laplacian $A=-\Delta$ with Dirichlet boundary condition, for some $\alpha>0$ and $1 \leqslant \rho<\frac{n+2}{n-2}$, with (H1), (H2) uniformly for $t \in \mathbb{R}$ and $\varepsilon \in[0,1]$ and (H3) uniformly for $\varepsilon \in[0,1]$.

In Section 2 we present a brief summary about the theory of autonomous and non-autonomous systems, with their respective attractors. In Section 3 we will describe the precise spaces, along with the required topologies, to fit the family of non-autonomous non-classical equations in the framework described in Subsection 1.1. Sections 4 and 5 are devoted to prove that each equation (6) generates a non-autonomous dynamical system and prove the existence of the several types of attractors described in Section 2, respectively.

In Sections 6 and 7, motivated by the discussion in Subsection 1.2, we study the upper semicontinuity and topological structural stability of each kind of global attractor found in Section 5, respectively. With all this work, we provide a complete study for the various scenarios of asymptotic behavior for non-autonomous dynamical systems described in Subsection 1.1.

\section{Preliminaries: Asymptotic dynamics of non-autonomous equations}

We will briefly present the theory described in [7], which studies non-autonomous differential equations in different frameworks and gives relations between these dynamics.

\subsection{Semigroups}

First of all, we define the notions of semigroups and their global attractors (the reader may see [18] for more details of this theory).

Let $(X, d)$ be a metric space and $\mathscr{C}(X)$ the set of all continuous maps from $X$ into itself. A semigroup in $X$ is a one parameter family $\{T(t): t \geqslant 0\}$ such that

(a) $T(0)=I d_{X}$, with $I_{X}$ being the identity in $X$,

(b) $T(t) T(s)=T(t+s)$, for all $t, s \geqslant 0$ and

(c) the map $[0, \infty) \times X \ni(t, x) \mapsto T(t) x \in X$ is continuous.

From now on we are going to denote by $\mathrm{d}_{\mathrm{H}}(\cdot, \cdot)$ the Hausdorff semidistance between two subsets of $X$, that is, for any $A, B \subset X$ :

$$
\mathrm{d}_{H}(A, B)=\sup _{a \in A} \inf _{b \in B} d(a, b) .
$$

Definition 1. A compact set $\mathscr{A}$ is called a global attractor of $\{T(t): t \geqslant 0\}$ if satisfies:

(i) $\mathscr{A}$ is invariant for $\{T(t): t \geqslant 0\}$; that is, $T(t) \mathscr{A}=\mathscr{A}$, for all $t \geqslant 0$.

(ii) $\mathscr{A}$ attracts bounded subsets under the action of $\{T(t): t \geqslant 0\}$; that is, for each bounded subset $B$ of $X$ we have

$$
\lim _{t \rightarrow \infty} \mathrm{d}_{H}(T(t) B, \mathscr{A})=0 .
$$

The global attractor of a semigroup describes the asymptotic behavior of the semigroup. To be more precise, we define a global solution of $\{T(t): t \geqslant 0\}$ as a function $\xi: \mathbb{R} \rightarrow X$ such that $T(t) \xi(s)=\xi(t+s)$ for all $t \geqslant 0$ and $s \in \mathbb{R}$. Then, we know that if $\mathscr{A}$ is the global attractor of $\{T(t): t \geqslant 0\}$ we have

$$
\mathscr{A}=\{x \in X: x=\xi(0) \text { for some bounded global solution } \xi \text { of }\{T(t): t \geqslant 0\}\} .
$$


This characterization means that not only the global attractor attracts all positive orbits $\{T(t) x: t \geqslant 0\}$ $(x \in X)$ but it actually consists of all bounded globally defined solutions. Moreover, the global attractor for a semigroup is unique.

To obtain existence of global attractors for semigroups, we will need some definitions.

Definition 2. Let $B, C \subset X$. We say that $B$ absorbs $C$ under the action of $\{T(t): t \geqslant 0\}$ if there exists $T \geqslant 0$ such that

$$
T(t) C \subset B, \text { for all } t \geqslant T .
$$

Definition 3. We say that a semigroup $\{T(t): t \geqslant 0\}$ is asymptotically compact if given sequences $t_{n} \rightarrow \infty$ and $\left\{x_{n}\right\}_{n \in \mathbb{B}}$ bounded in $X$ such that $\left\{T\left(t_{n}\right) x_{n}\right\}_{n \in \mathbb{N}}$ is bounded, then $\left\{T\left(t_{n}\right) x_{n}\right\}_{n \in \mathbb{N}}$ is precompact in $X$.

With these definitions we are able to state the main result about existence of global attractors, that we be needed later.

Theorem 1 (Theorem 3.4 in [18]). Let $\{T(t): t \geqslant 0\}$ be an asymptotically compact semigroup. Assume that there exists a bounded set $B \subset X$ such that $B$ absorbs all bounded subsets of $X$ under the action of $\{T(t): t \geqslant 0\}$. Then $\{T(t): t \geqslant 0\}$ has a global attractor $\mathscr{A}$ and $\mathscr{A} \subset B$.

\subsection{Evolutions processes}

Now we are going to define evolution processes and their pullback atractors (see $[9,16]$ for more details). These concepts appear in the literature as natural generalizations for semigroups and global attractors, respectively.

Again, let $(X, d)$ be a metric space. An evolution process in $X$ is a two parameter family $\{T(t, s): t \geqslant s\}$ in $\mathscr{C}(X)$ such that

(a) $T(t, t)=I d_{X}$,

(b) $T(t, s) T(s, \tau)=T(t, \tau)$, for all $t \geqslant s \geqslant \tau$ and

(c) the map $\mathscr{P} \times X \ni(t, s, x) \mapsto T(t, s) x \in X$ is continuous, where $\mathscr{P}=\left\{(t, s) \in \mathbb{R}^{2}: t \geqslant s\right\}$.

Definition 4. A family of compact sets $\{A(t)\}_{t \in \mathbb{R}}$ is called a pullback attractor of $T(t, s)$ if satisfies:

(i) $\{A(t)\}_{t \in \mathbb{R}}$ is invariant; that is, $T(t, s) A(s)=A(t)$, for all $t \geq s$.

(ii) $\{A(t)\}_{t \in \mathbb{R}}$ pullback attracts bounded subsets; that is, for each bounded subset $B$ of $X$ and $t \in \mathbb{R}$, we have

$$
\lim _{s \rightarrow-\infty} \mathrm{d}_{H}(T(t, s) B, A(t))=0
$$

(iii) $\{A(t)\}_{t \in \mathbb{R}}$ is the minimal family of closed sets with property (ii).

Remark 4. We note that when $T(t, s)=S(t-s)$, the family $\{S(t): t \geqslant 0\}$ is a semigroup in $X$ and Definition 4 reduces to the definition of global attractors. The first difference that appears is item (iii) in Definition 4 and it ensures the uniqueness of the pullback attractor, since it does not follows directly from (i) and (ii) as in the autonomous case.

\subsection{Non-autonomous dynamical systems}

Now, we will introduce the concept of non- autonomous dynamical systems, which is a general method that provides a way to form the base space for a given non-autonomous differential equation. The idea of this method is to consider the family of non-linearities as a base flow driven by the time shift. 
Definition 5. A non-autonomous dynamical system (NDS) is a quadruple $(\varphi, \theta)_{(X, \Sigma)}$, where $X, \Sigma$ are a metric spaces with metrics $d_{X}$ and $d_{\Sigma}$, respectively; $\theta \doteq\left\{\theta_{t}: t \geqslant 0\right\}$ is a semigroup in $\Sigma$, called the shift operator (or driving semigroup), and $\varphi: \mathbb{R}^{+} \times \Sigma \times X \rightarrow X$ is a map ${ }^{\mathrm{c}}$ that verifies

(i) $\varphi(0, \sigma)=I d_{X}$ for all $\sigma \in \Sigma$;

(ii) $\mathbb{R}^{+} \times \Sigma \ni(t, \sigma) \mapsto \varphi(t, \sigma) u \in X$ is continuous, and

(iii) $\varphi(t+s, \sigma)=\varphi\left(t, \theta_{s} \sigma\right) \varphi(s, \sigma)$, for all $t, s \geqslant 0$ and $\sigma \in \Sigma$.

The map $\varphi$ is called the cocycle semiflow and property (iii) is know as the cocycle property.

To define the cocycle attractor and the uniform attractor for a NDS $(\varphi, \theta)_{(X, \Sigma)}$, we first must define the concepts of non-autonomous set, invariance and pullback attraction in this framework:

Definition 6. A non-autonomous set is a family $\{D(\sigma)\}_{\sigma \in \Sigma}$ of subsets of $X$ indexed in $\Sigma$. We say that $\{D(\sigma)\}_{\sigma \in \Sigma}$ is an open (closed, compact) non-autonomous set if each fiber $D(\sigma)$ is an open (closed, compact) subset of $X$.

Definition 7. A non-autonomous set $\{D(\sigma)\}_{\sigma \in \Sigma}$ is invariant under the NDS $(\varphi, \theta)_{(X, \Sigma)}$ if

$$
\varphi(t, \sigma) D(\sigma)=D\left(\theta_{t} \sigma\right), \text { for all } t \geqslant 0 \text { and } \sigma \in \Sigma \text {. }
$$

To define the concept of pullback attraction, we must ask some additional properties on $\Sigma$, and from now on, we are going to assume that $\Sigma$ is compact and invariant for the driving semigroup $\left\{\theta_{t}: t \geqslant 0\right\}$, and also that $\left\{\theta_{t}: t \geqslant 0\right\}$ is a group over $\Sigma$; that is, $\theta_{t}$ is invertible, and we denote $\theta_{t}^{-1}=\theta_{-t}$.

Remark 5. Actually, these assumptions can be dropped. We can obtain the same results requiring only that $\left\{\theta_{t}: t \geqslant 0\right\}$ possess a global attractor in $\Sigma$, with virtually no additional work, but with a more difficult notation. So, for simplicity, we shall assume all the hypotheses above.

Definition 8. A compact non-autonomous set $\{A(\sigma)\}_{\sigma \in \Sigma}$ is called a cocycle attractor of $(\varphi, \theta)_{(X, \Sigma)}$ if

(i) $\{A(\sigma)\}_{\sigma \in \Sigma}$ is invariant under $(\varphi, \theta)_{(X, \Sigma)}$;

(ii) $\{A(\sigma)\}_{\sigma \in \Sigma}$ pullback attracts all bounded subsets $B \subset X$, i.e.

$$
\lim _{t \rightarrow+\infty} \mathrm{d}_{H}\left(\varphi\left(t, \theta_{-t} \sigma\right) B, A(\sigma)\right)=0 .
$$

(iii) $\{A(\sigma)\}_{\sigma \in \Sigma}$ is the minimal among the closed non-autonomous sets with property (ii).

We can also deal with the uniform attraction for a NDS - in this framework, the attraction do not depend on the chosen $\sigma \in \Sigma$ - that is, we say that the subset $K \subseteq X$ is uniform attracting for the $\operatorname{NDS}(\varphi, \sigma)_{(X, \Sigma)}$ if for each $B \subset X$ bounded,

$$
\lim _{t \rightarrow \infty} \sup _{\sigma \in \Sigma} \mathrm{d}_{H}(\varphi(t, \sigma) B, K)=0 .
$$

Definition 9. A compact subset $\mathscr{A} \subset X$ is called the uniform attractor for the NDS $(\varphi, \sigma)_{(X, \Sigma)}$ if it is the minimal closed subset of $X$ that uniform attracts all bounded subsets of $X$.

Theorem 2. A NDS $(\varphi, \sigma)_{(X, \Sigma)}$ has a uniform attractor if and only if there exists a compact uniform attracting set $K$.

${ }^{\mathrm{c}}$ Note that we use the notation $\varphi(t, \sigma, x)=\varphi(t, \sigma) x$ for all $(t, \sigma, x) \in \mathbb{R}^{+} \times \Sigma \times X$. 


\subsection{Skew-product semiflows}

When dealing with non-autonomous dynamical systems, it is worthwhile to question if we can transform them into an autonomous one; and that is precisely the case: for a given NDS $(\varphi, \theta)_{(X, \Sigma)}$ we can define a semigroup $\{\Pi(t): t \geqslant 0\}$ in the product space $\mathbb{X}=X \times \Sigma$ as (see [27,28] for more details)

$$
\Pi(t)(u, \sigma)=\left(\varphi(t, \sigma) u, \theta_{t} \sigma\right),
$$

which is called skew-product semiflow associated with $(\varphi, \theta)_{(X, \Sigma)}$.

So far we obtained three different objects:

1. the evolution process $\{T(t, s): t \geqslant s\}$;

2. the non-autonomous dynamical system $(\varphi, \theta)_{(X, \Sigma)}$ and

3. the skew-product semiflow $\{\Pi(t): t \geqslant 0\}$,

and the four different notions of 'attractors' listed in the Introduction:

(i) the pullback attractor $\{A(t)\}_{t \in \mathbb{R}}$ of $\{T(t, s): t \geqslant s\}$;

(ii) the cocycle attractor $\{A(\sigma)\}_{\sigma \in \Sigma}$ of $(\varphi, \theta)_{(X, \Sigma)}$;

(iii) the uniform attractor $\mathscr{A}$ of $(\varphi, \theta)_{(X, \Sigma)}$ and

(iv) the global attractor $\mathbb{A}$ of $\{\Pi(t): t \geqslant 0\}$,

and now we presente briefly the relationships between these objects (as in [7]). We begin with the relation between the global attractor of $\{\Pi(t): t \geqslant 0\}$ and the cocycle attractor of $(\varphi, \theta)_{(X, \Sigma)}$.

Theorem 3 (Propositions 3.30 and 3.31 in [22], or Theorem 3.4 in [14]). Let $(\varphi, \theta)_{(X, \Sigma)}$ be a non-autonomous dynamical system and let $\{\Pi(t): t \geqslant 0\}$ be the associated skew product semiflow on $X \times \Sigma$ with a global attractor $\mathbb{A}$. Then $\{A(\sigma)\}_{\sigma \in \Sigma}$ with $A(\sigma)=\{x \in X:(x, \sigma) \in \mathbb{A}\}$ is the cocycle attractor of $(\varphi, \theta)_{(X, \Sigma)}$.

The following theorem shows the relationship between the global attractor of a skew product semiflow and the pullback attractors of the evolution processes it may contain.

Theorem 4 (Theorem 2.7 in [5]). Assume that the skew product semiflow $\{\Pi(t): t \geqslant 0\}$ possesses a global attractor $\mathbb{A}$. Then the evolution process $\left\{T_{\sigma}(t, s): t \geqslant s\right\}$ given by

$$
T_{\sigma}(t, s) u=\varphi\left(t-s, \theta_{s} \sigma\right) u, u \in X,
$$

possesses a pullback attractor $\left\{A_{\sigma}(t)\right\}_{t \in \mathbb{R}}$. Moreover,

$$
\mathbb{A}=\bigcup_{\sigma \in \Sigma}\left[\bigcup_{t \in \mathbb{R}} A_{\sigma}(t) \times\{\sigma\}\right] .
$$

Therefore, if $\mathbb{A}$ is the global attractor of the associated semigroup $\{\Pi(t): t \in \mathbb{R}\}$ of $(\varphi, \sigma)_{(X, \Sigma)}$, then the uniform attractor $\mathscr{A}$ is the projection on $X$ of the global attractor, that is, $\mathscr{A}=\pi_{X}(\mathbb{A})$, where $\pi_{X}: X \times \Sigma \rightarrow X$ is the projection over $X$.

Now, the relationship between the uniform attractor and the pullback attractor is clear.

Theorem 5. The $\operatorname{NDS}(\varphi, \sigma)_{(X, \Sigma)}$ has a uniform attractor $\mathscr{A}$ if and only if the associated skew-product semiflow $\{\Pi(t): t \geqslant 0\}$ has a global attractor $\mathbb{A}$ and

$$
\mathscr{A}=\pi_{X}(\mathbb{A})=\bigcup_{\sigma \in \Sigma} \bigcup_{t \in \mathbb{R}} A_{\sigma}(t)
$$


The following result shows us the required assumptions in order to obtain the existence of the global attractor for the skew-product semiflow $\{\Pi(t): t \geqslant 0\}$ associated with the NDS $(\varphi, \sigma)_{(X, \Sigma)}$ based on the existence of its cocycle attractor (see $[14,22]$ for more details).

Theorem 6. Suppose that $\{A(\sigma)\}_{\sigma \in \Sigma}$ is the cocycle attractor of $(\varphi, \theta)_{(X, \Sigma)},\{\Pi(t): t \geqslant 0\}$ is the associated skew-product semiflow. Assume that $\{A(\sigma)\}_{\sigma \in \Sigma}$ is uniformly attracting, i.e.,

$$
\lim _{t \rightarrow+\infty} \sup _{\sigma \in \Sigma} \operatorname{dist}\left(\varphi\left(t, \theta_{-t} \sigma\right) D, A(\sigma)\right)=0,
$$

and that $\bigcup_{\sigma \in \Sigma} A(\sigma)$ is precompact in $X$. Then the set $\mathbb{A}$ associated with $\{A(\sigma)\}_{\sigma \in \Sigma}$, given by

$$
\mathbb{A}=\bigcup_{\sigma \in \Sigma} A(\sigma) \times\{\sigma\}
$$

is the global attractor of $\{\Pi(t): t \geqslant 0\}$.

With these results we complete the relations between the four different asymptotic dynamics we presented. In this paper, as we said before, we will try to deal with these four dynamics, to obtain as much information as we can of equation (6).

\section{Driving semigroups of translations for (6)}

In this section we will put the family of equations (6) in the framework described on Subsection 1.1. To this end, let $A=-\Delta: D(A) \subset X \rightarrow X$ be the negative Laplacian operator with Dirichlet boundary condition, defined in $D(A)=H_{0}^{1}(\Omega) \cap H^{2}(\Omega)$, where $X=L^{2}(\Omega)$. Consider the fractional power scale $X^{\alpha}$, with $\alpha \in \mathbb{R}$, generated by $(X, A)$. Also, consider the Nemytskii operators $g_{\varepsilon}^{e}(\cdot, \cdot)$ defined as $g_{\varepsilon}^{e}(t, u)(x)=g_{\varepsilon}(t, u(x))$ for each $(t, x) \in \mathbb{R} \times \Omega$ and $u: \Omega \rightarrow \mathbb{R}$.

Following the ideas in [26], we define the operators

$$
B_{\gamma}(t)=(I+\gamma(t) A)^{-1} \text { and } \tilde{A}_{\gamma}(t)=A B_{\gamma}(t),
$$

and the function $g_{\varepsilon, \gamma}(t, u)=B_{\gamma}(t) g_{\varepsilon}^{e}(t, u)$, for each $\varepsilon \in[0,1]$, we can write problem (6) as

$$
u_{t}=F_{\varepsilon}(t, u)
$$

where $F_{\varepsilon}(t, u)=-\tilde{A}_{\gamma}(t) u+g_{\varepsilon, \gamma}(t, u)$. The domain of the operators $\tilde{A}_{\gamma}(t)$ does not depend on time and the operators $\mathbb{R} \ni t \mapsto B_{\gamma}(t)$ and $\mathbb{R} \ni t \mapsto \tilde{A}_{\gamma}(t)$ are absolutely continuous functions.

To study (6), for each $\varepsilon \in[0,1]$, we must be able to study the hull of the function $F_{\varepsilon}(\cdot, \cdot)$ in a suitable space. This is our task in the next subsection.

\subsection{Driving groups of translations}

We begin considering the sets

- $\mathscr{C}_{1}=C_{b}(\mathbb{R}, \mathbb{R})$ of continuous bounded functions from $\mathbb{R}$ to itself with metric

$$
d_{1}\left(\lambda_{1}, \lambda_{2}\right)=\sup _{t \in \mathbb{R}}\left|\lambda_{1}(t)-\lambda_{2}(t)\right|
$$

- $\mathscr{C}_{2}$ of continuous functions from $\mathbb{R}^{2}$ to $\mathbb{R}$, which satisfies: $h \in \mathscr{C}_{2}$ if there exists constants $\gamma, \omega \geqslant 0$ such that

$$
\sup _{t \in \mathbb{R}}\left|h\left(t, s_{1}\right)-h\left(t, s_{2}\right)\right| \leqslant \gamma\left|s_{1}-s_{2}\right|\left(1+\left|s_{1}\right|^{\rho-1}+\left|s_{2}\right|^{\rho-1}\right), \text { for all } s_{1}, s_{2} \in \mathbb{R},
$$


and

$$
\sup _{t \in \mathbb{R}}|h(t, 0)| \leqslant \omega .
$$

In $\mathscr{C}_{2}$ we introduce the norm

$$
\|h\|_{\mathscr{C}_{2}}=\sup _{\substack{t, s \in \mathbb{R} \\ s \neq 0}} \frac{|h(t, s)-h(t, 0)|}{|s|\left(1+|s|^{\rho-1}\right)}+\sup _{t \in \mathbb{R}}|h(t, 0)|,
$$

and the distance

$$
d_{2}\left(h_{1}, h_{2}\right)=\left\|h_{1}-h_{2}\right\|_{\mathscr{C}_{2}}
$$

Remark 6. We have that $\mathscr{C}_{1}$ and $\mathscr{C}_{2}$ are Banach spaces with norms $\|\lambda\|_{\mathscr{C}_{1}}=\sup _{t \in \mathbb{R}}|\lambda(t)|$ and $\|\cdot\|_{\mathscr{C}_{2}}$, respectively.

Clearly we have that $\gamma \in \mathscr{C}_{1}$ and $g_{\varepsilon} \in \mathscr{C}_{2}$, for all $\varepsilon \in[0,1]$. We define the group of translations in both ${ }^{\mathrm{d}} \mathscr{C}_{1}$ and $\mathscr{C}_{2},\left\{\theta_{t}: t \in \mathbb{R}\right\}$, by

$$
\theta_{t} \lambda(s)=\lambda(t+s), \text { for all } \lambda \in \mathscr{C}_{1} \text { and } t, s \in \mathbb{R}
$$

and

$$
\theta_{t} h(r, s)=h(t+r, s), \text { for all } h \in \mathscr{C}_{2} \text { and } t, r, s \in \mathbb{R} .
$$

Also, let

- $\Gamma$ be the hull of $\gamma$ in $\mathscr{C}_{1}$; that is,

$$
\Gamma=\text { closure of }\left\{\theta_{t} \gamma\right\}_{t \in \mathbb{R}} \text { in }\left(\mathscr{C}_{1}, d_{1}\right) .
$$

- $\mathscr{G}_{\varepsilon}$ be the hull of $g_{\varepsilon}$ in $\mathscr{C}_{2}$; that is,

$$
\mathscr{G}_{\varepsilon}=\text { closure of }\left\{\theta_{t} g_{\varepsilon}\right\}_{t \in \mathbb{R}} \text { in }\left(\mathscr{C}_{2}, d_{2}\right) .
$$

\section{Remark 7.}

1. Since $\gamma$ is bounded and uniformly continuous on $\mathbb{R}$, the set $\Gamma$ is compact in $\left(\mathscr{C}_{1}, d_{1}\right)$, using the ArzeláAscoli Theorem.

2. Note that, by simple computations, we have that there exists a constant $C>0$ such that

$$
\sup _{t \in \mathbb{R}}\left\|B_{\lambda_{1}}(t)-B_{\lambda_{2}}(t)\right\|_{\mathscr{L}\left(H^{-1}, H_{0}^{1}(\Omega)\right)} \leqslant C\left\|\lambda_{1}-\lambda_{2}\right\|_{\mathscr{C}_{1}}
$$

and

$$
\sup _{t \in \mathbb{R}}\left\|\tilde{A}_{\lambda_{1}}(t)-\tilde{A}_{\lambda_{2}}(t)\right\|_{\mathscr{L}\left(H_{0}^{1}(\Omega)\right)} \leqslant C\left\|\lambda_{1}-\lambda_{2}\right\|_{\mathscr{C}_{1}},
$$

for all $\lambda_{1}, \lambda_{2} \in \Gamma$, where $H^{-1}$ is the dual space of $H_{0}^{1}(\Omega)$.

3. Since $g_{0}(t, s)=f(s)$ for all $t, s \in \mathbb{R}, \mathscr{G}_{0}=\{f\}$ and hence it is compact in $\left(\mathscr{C}_{2}, d_{2}\right)$.

4. Since hypotheses (H1)-(H4) are uniform for $t \in \mathbb{R}$, they all are satisfied by every function in $\mathscr{G}_{\varepsilon}$.

Using items 1 and 2 from the previous remark, we will make one additional assumption on the family $\left\{g_{\varepsilon}\right\}$ :

$$
\mathscr{G}_{\varepsilon} \text { is compact in }\left(\mathscr{C}_{2}, d_{2}\right) \text { for each } \varepsilon \in(0,1] \text {. }
$$

\footnotetext{
${ }^{\mathrm{d}}$ Here we denote both groups the same, since there will be no confusion of notation.
} 
Remark 8. Condition $(\mathbf{C})$ is verified, for instance, when each $g_{\varepsilon}$ is time-independent, or periodic in $t$, or almostperiodic in $t$ (for the latter, see for instance [20, Appendix - Theorem 11]).

Now we can see that each function $h_{\varepsilon} \in \mathscr{G}_{\varepsilon}$ defines a Nemytskii operator from $\mathbb{R} \times X^{\frac{s}{2}}$ into $L^{\frac{2 n}{n+2 r}}(\Omega)$, for suitable $s$ and $r$.

Lemma 7. Assume that the family $\left\{g_{\varepsilon}\right\}_{\varepsilon \in[0,1]}$ satisfies (H1), $A$ is the negative Dirichlet Laplacian in $X$ with domain $X^{1}=H^{2}(\Omega) \cap H_{0}^{1}(\Omega)$ and consider its closed extension to $H^{-r}=\left(X^{\frac{r}{2}}\right)^{\prime}$, the dual space of $X^{\frac{r}{2}}$, (in particular, $\left.H^{-1}=H_{0}^{1}(\Omega)^{\prime}\right)$. Then the Nemytskii operators $\left\{h_{\varepsilon}^{e}\right\}_{\varepsilon \in[0,1]}$ are well defined from $\mathbb{R} \times X^{\frac{s}{2}}$ into $L^{\frac{2 n}{n+2 r}}(\Omega)$, provided that $r \in\left[\frac{(\rho-1)(n-2)}{4}, 1\right], s \in[r, 1] \cap\left[\frac{n}{2}-\frac{2}{\rho-1}, 1\right]$, for each $h_{\varepsilon} \in \mathscr{G}_{\varepsilon}$. If $B$ is a bounded subset of $X^{\frac{s}{2}}$ then there exists a constant $C=C(B)>0$ such that

$$
\sup _{t \in \mathbb{R}}\left\|h_{\varepsilon}^{e}\left(t, u_{1}\right)-h_{\varepsilon}^{e}\left(t, u_{2}\right)\right\|_{L^{\frac{2 n}{n+2 r}}(\Omega)} \leqslant C\left\|u_{1}-u_{2}\right\|_{X^{\frac{s}{2}}}, \text { for all } \varepsilon \in[0,1] .
$$

Moreover, if $r$ can be taken strictly less than 1 and $J \subset \mathbb{R}$ is an arbitrary subset, $h_{\varepsilon}^{e}$ takes $J \times B$ in a precompact set of $H^{-1}$, for each $\varepsilon \in[0,1]$.

Proof. Following [10], using hypothesis (H1) we have that

$$
\begin{aligned}
\sup _{t \in \mathbb{R}} \| h_{\varepsilon}^{e}(t, u)-h_{\varepsilon}^{e}(t, v) & \|_{L^{\frac{2 n}{n+2 r}(\Omega)}} \leqslant c\left[\int_{\Omega}\left[|u-v|\left(1+|u|^{\rho-1}+|v|^{\rho-1}\right)\right]^{\frac{2 n}{n+2 r}}\right]^{\frac{n+2 r}{2 n}} \\
& \leqslant \tilde{c}\|u-v\|_{L^{\frac{2 n}{n-2 r}(\Omega)}}\left(1+\|u\|_{L^{\frac{n(\rho-1)}{2 r}(\Omega)}}^{\rho-1}+\|v\|_{L^{\frac{n(\rho-1)}{2 r}(\Omega)}}^{\rho-1}\right) \\
& \leqslant \bar{c}\|u-v\|_{X^{\frac{s}{2}}}\left(1+\|u\|_{X^{\frac{s}{2}}}^{\rho-1}+\|v\|_{X^{\frac{s}{2}}}^{\rho-1}\right) .
\end{aligned}
$$

or any $s \in[r, 1] \cap\left[\frac{n}{2}-\frac{2}{\rho-1}, 1\right]$. The last statement holds since $H^{-r}$ is compact embedded in $H^{-1}$ and $L^{\frac{2 n}{n+2 r}}(\Omega) \hookrightarrow$ $H^{-r}$ for $r<1$.

We define now

- $\mathscr{C}_{2}^{e}=C_{b}^{0}\left(\mathbb{R} \times H_{0}^{1}(\Omega), L^{\frac{2 n}{n+2}}(\Omega)\right)$ as the set of all continuous functions from $\mathbb{R} \times H_{0}^{1}(\Omega)$ taking values on $L^{\frac{2 n}{n+2}}(\Omega)$ that are bounded on sets $\mathbb{R} \times B$, where $B$ is a bounded set of $H_{0}^{1}(\Omega)$ with metric

$$
d_{2}^{e}\left(\sigma_{1}, \sigma_{2}\right)=\sum_{k=1}^{\infty} 2^{-k} \frac{\left\|\sigma_{1}-\sigma_{2}\right\|_{k}^{e}}{1+\left\|\sigma_{1}-\sigma_{2}\right\|_{k}^{e}},
$$

where if $B^{k}=\left\{u \in H_{0}^{1}(\Omega):\|u\|_{H_{0}^{1}(\Omega)} \leqslant k\right\}$ we have

$$
\left\|\sigma_{1}-\sigma_{2}\right\|_{k}^{e}=\sup _{t \in \mathbb{R}} \sup _{u \in B^{k}}\left\|\sigma_{1}(t, u)-\sigma_{2}(t, u)\right\|_{L^{\frac{2 n}{n+2}}(\Omega)} .
$$

Remark 9. Here we have that $\mathscr{C}_{2}^{e}$ with the metric $d_{2}^{e}$ is a Frechét space, and a sequence $\left\{\sigma_{k}\right\}_{k \in \mathbb{N}}$ converges in $C_{2}^{e}$ if and only if it converges in each seminorm $\|\cdot\|_{n}^{e}$.

Define the group of translations ${ }^{\mathrm{e}}\left\{\theta_{t}: t \in \mathbb{R}\right\}$ in $\mathscr{C}_{2}^{e}$ by

$$
\theta_{t} h(s, v)=h(t+s, v), \text { for all } h \in \mathscr{C}_{2}^{e}, t, s \in \mathbb{R} \text { and } v \in H_{0}^{1}(\Omega) .
$$

Since Lemma 7 implies that $g_{\varepsilon}^{e} \in \mathscr{C}_{2}^{e}$, for all $\varepsilon \in[0,1]$, let also

\footnotetext{
e Again, since there will be no confusion, we denote the group of translations the same.
} 
- $\mathscr{G}_{\varepsilon}^{e}$ be the hull of $g_{\varepsilon}^{e}$ in $\mathscr{C}_{2}^{e}$; that is,

$$
\mathscr{G}_{\varepsilon}^{e}=\text { closure of }\left\{\theta_{t} g_{\varepsilon}^{e}\right\}_{t \in \mathbb{R}} \text { in }\left(\mathscr{C}_{2}^{e}, d_{2}^{e}\right) .
$$

Now, in order to have a better understanding of the set $\mathscr{G}_{\varepsilon}^{e}$, we present the following results:

Lemma 8. If $h \in \mathscr{G}_{\mathcal{E}}$, then $h^{e} \in \mathscr{G}_{\varepsilon}^{e}$.

Proof. This follows easily by Lemma 7.

Lemma 9. There exists a constant $L \geqslant 0$, such that for all $h_{1}, h_{2} \in \mathscr{G}_{\varepsilon}$, we have that

$$
d_{2}^{e}\left(h_{1}^{e}, h_{2}^{e}\right) \leqslant L\left\|h_{1}-h_{2}\right\|_{\mathscr{C}_{2}}
$$

Proof. Fix $k \in \mathbb{N}$. We have that

$$
\begin{aligned}
& \left\|h_{1}^{e}-h_{2}^{e}\right\|_{k}^{e}=\sup _{t \in \mathbb{R}} \sup _{u \in B^{k}}\left\|h_{1}^{e}(t, u)-h_{2}^{e}(t, u)\right\|_{L^{\frac{2 n}{n+2}}(\Omega)} \\
& \quad=\sup _{t \in \mathbb{R}} \sup _{u \in B^{k}}\left(\int_{\Omega}\left|h_{1}(t, u(x))-h_{2}(t, u(x))\right|^{\frac{2 n}{n+2}} d x\right)^{\frac{n+2}{2 n}} \\
& \quad \leqslant \tilde{c}\left\|h_{1}-h_{2}\right\|_{\mathscr{C}_{2}} \cdot \sup _{u \in B^{k}}\left(\int_{\Omega}\left|u(x)\left(1+|u(x)|^{\rho-1}\right)\right|^{\frac{2 n}{n+2}} d x\right)^{\frac{n+2}{2 n}},
\end{aligned}
$$

where $\tilde{c}$ does not depend on $h_{1}, h_{2}$ and hence, arguing as in Lemma 7 , we obtain

$$
\begin{aligned}
\left\|h_{1}^{e}-h_{2}^{e}\right\|_{k}^{e} & \leqslant \bar{c}\left\|h_{1}-h_{2}\right\|_{\mathscr{C}_{2}} \cdot \sup _{u \in B^{k}}\left[\|u\|_{H_{0}^{1}(\Omega)}\left(1+\|u\|_{H_{0}^{1}(\Omega)}^{\rho-1}\right)\right] \\
& \leqslant \hat{c}\left\|h_{1}-h_{2}\right\|_{\mathscr{C}_{2}}\left[k\left(1+k^{\rho-1}\right)\right],
\end{aligned}
$$

where $\hat{c}$ does not depend on $h_{1}, h_{2}$.

Hence

$$
d_{2}^{e}\left(h_{1}^{e}, h_{2}^{e}\right) \leqslant \hat{c}\left\|h_{1}-h_{2}\right\|_{\mathscr{C}_{2}} \sum_{k=1}^{\infty} 2^{-k+1} k^{\rho}
$$

and the result follows since $\sum_{k=1}^{\infty} 2^{-k+1} k^{\rho}$ is a convergent series.

Proposition 10. If condition (C) holds then $\sigma_{\varepsilon} \in \mathscr{G}_{\varepsilon}^{e}$ if and only if there exists $h_{\varepsilon} \in \mathscr{G}_{\varepsilon}$ such that $\sigma_{\varepsilon}=h_{\varepsilon}^{e}$, for each $\varepsilon \in[0,1]$.

Proof. The result is trivial if $\varepsilon=0$. Assume that $\varepsilon \in(0,1]$. One inclusion follows from Lemma 8. Now if $\sigma_{\varepsilon} \in \mathscr{G}_{\varepsilon}^{e}$, then exists a sequence $\left\{t_{n}\right\}_{n \in \mathbb{N}} \subset \mathbb{R}$ such that $\theta_{t_{n}} g_{\varepsilon}^{e} \rightarrow \sigma_{\varepsilon}$ in $\mathscr{C}_{2}^{e}$, by definition. Consider the sequence $\left\{\theta_{t_{n}} g_{\varepsilon}\right\}_{n \in \mathbb{N}}$ in $\mathscr{C}_{2}$. Since $\mathscr{G}_{\varepsilon}$ is compact, we can assume without loss of generality, that $\theta_{t_{n}} g_{\varepsilon} \rightarrow h_{\varepsilon} \in \mathscr{G}_{\varepsilon}$ in $\mathscr{C}_{2}$. Thus, using Lemma 9 we have that

$$
\begin{aligned}
d_{2}^{e}\left(\sigma_{\varepsilon}, h_{\varepsilon}^{e}\right) & \leqslant d_{2}^{e}\left(\sigma_{\varepsilon}, \theta_{t_{n}} g_{\varepsilon}^{e}\right)+d_{2}^{e}\left(\theta_{t_{n}} g_{\varepsilon}^{e}, h_{\varepsilon}^{e}\right) \\
& \leqslant d_{2}^{e}\left(\sigma_{\varepsilon}, \theta_{t_{n}} g_{\varepsilon}^{e}\right)+L\left\|\theta_{t_{n}} g_{\varepsilon}-h_{\varepsilon}\right\|_{\mathscr{C}_{2}},
\end{aligned}
$$

and making $n \rightarrow \infty$ we obtain that $\sigma_{\varepsilon}=h_{\varepsilon}^{e}$.

Corollary 11. If condition $(\mathbf{C})$ holds, then $\mathscr{G}_{\varepsilon}^{e}$ is compact in $\mathscr{C}_{2}^{e}$, for each $\varepsilon \in[0,1]$.

Now we are finally in condition to define the space that will be suitable for our study of (6). Define 
- $\mathscr{C}_{*}=C_{b}^{0}\left(\mathbb{R} \times H_{0}^{1}(\Omega), H_{0}^{1}(\Omega)\right)$ the set of continuous functions which are bounded in sets of the form $\mathbb{R} \times B$, where $B$ is a bounded subset of $H_{0}^{1}(\Omega)$, with distance defined as

$$
d_{*}\left(\sigma_{1}, \sigma_{2}\right)=\sum_{n=1}^{\infty} 2^{-n} \frac{\left\|\sigma_{1}-\sigma_{2}\right\|_{n}^{*}}{1+\left\|\sigma_{1}-\sigma_{2}\right\|_{n}^{*}},
$$

where if $B^{n}=\left\{u \in H_{0}^{1}(\Omega):\|u\|_{H_{0}^{1}(\Omega)} \leqslant n\right\}$ we have

$$
\left\|\sigma_{1}-\sigma_{2}\right\|_{n}^{*}=\sup _{t \in \mathbb{R}} \sup _{u \in B^{n}}\left\|\sigma_{1}(t, u)-\sigma_{2}(t, u)\right\|_{H_{0}^{1}(\Omega)} .
$$

Now let $F_{\varepsilon}(t, u)=-\tilde{A}_{\gamma}(t) u+B_{\gamma}(t) g^{e}(t, u)$ be given as in (8). It is simple to see, recalling the definitions of $\tilde{A}_{\gamma}(t)$ and $B_{\gamma}(t)$ and the fact that $g_{\varepsilon}^{e} \in \mathscr{C}_{2}^{e}$, that $F_{\varepsilon} \in \mathscr{C}_{*}$ for each $\varepsilon \in[0,1]$. Again, we can define the group ${ }^{\mathrm{a}}$ $\left\{\theta_{t}: t \in \mathbb{R}\right\}$ in $\mathscr{C}_{*}$ by

$$
\theta_{t} h(s, v)=h(t+s, v), \text { for all } h \in \mathscr{C}_{*}, t, s \in \mathbb{R} \text { and } v \in H_{0}^{1}(\Omega) .
$$

Definition 10. With the notations above, we set $\Sigma_{\varepsilon}$ as the hull of $F_{\varepsilon}$ in $\mathscr{C}_{*}$; that is,

$$
\Sigma_{\varepsilon}=\text { closure of }\left\{\theta_{t} F_{\varepsilon}\right\}_{t \in \mathbb{R}} \text { in }\left(\mathscr{C}_{*}, d_{*}\right) .
$$

Before we proceed with the study of (6), we will need some characterization result for $\Sigma_{\varepsilon}$.

Lemma 12. We have that

(a) $\Sigma_{0}=\left\{B_{\lambda} f^{e}-\tilde{A}_{\lambda}\right\}_{\lambda \in \Gamma}$ and it is compact in $\left(\mathscr{C}_{*}, d_{*}\right)$;

(b) if (C) holds true, then for each $\varepsilon>0$ we have

$$
\Sigma_{\varepsilon} \subseteq\left\{B_{\lambda} h_{\varepsilon}^{e}-\tilde{A}_{\lambda}\right\}_{\lambda \in \Gamma, h \varepsilon \in \mathscr{G}_{\varepsilon}},
$$

and $\Sigma_{\varepsilon}$ is compact in $\left(\mathscr{C}_{*}, d_{*}\right)$.

where $B_{\lambda}(t)=(I+\lambda(t) A)^{-1}$ and $\tilde{A}_{\lambda}(t)=A B_{\lambda}(t)$, for each $\lambda \in \Gamma$.

Proof. Since $g_{0}(t, s)=f(s)$ for all $t, s \in \mathbb{R}$ and $\Gamma$ is compact, item (a) follows immediately. Now, fix $\varepsilon>0$ and let $H_{\varepsilon} \in \Sigma_{\varepsilon}$. Then, by definition, there exists a real sequence $\left\{t_{n}\right\}_{n \in \mathbb{N}}$ such that $H_{\varepsilon}=\mathscr{C}_{*}-\lim _{n \rightarrow \infty} \theta_{t_{n}}\left(B_{\gamma} g_{\varepsilon}^{e}-\tilde{A}_{\gamma}\right)$, that is, the sequence $\theta_{t_{n}}\left(B_{\gamma} g_{\varepsilon}^{e}-\tilde{A}_{\gamma}\right)$ converges to $H_{\varepsilon}$ in the metric of $\mathscr{C}_{*}$ defined above.

We can extract a subsequence of $\left\{t_{n}\right\}_{n \in \mathbb{N}}$, which we shall denote the same, and elements $\lambda \in \Gamma$ and $h_{\varepsilon} \in \mathscr{G}_{\varepsilon}$ such that $\gamma=\mathscr{C}-\lim _{n \rightarrow \infty} \theta_{s_{n}} \gamma$ and $h_{\varepsilon}^{e}=\mathscr{C}_{2}^{e}-\lim _{n \rightarrow \infty} \theta_{t_{n}} g_{\varepsilon}^{e}$, by the compactness of $\Gamma$ and $\mathscr{G}_{\varepsilon}$ and Proposition 10. Thus $H_{\varepsilon}=B_{\lambda} h_{\varepsilon}^{e}-\tilde{A}_{\lambda}$.

The last statement is clear from (a) and (b), which concludes the result.

Thus our problem in $H_{0}^{1}(\Omega)$ takes the form

$$
\left\{\begin{array}{l}
\dot{u}=H_{\mathcal{E}}(t, u), \quad t>0 \\
u(0)=u_{0} \in H_{0}^{1}(\Omega),
\end{array}\right.
$$

for each $H_{\varepsilon} \in \Sigma_{\varepsilon}$, which is precisely equation (4). As a matter of fact we have, for each fixed $\varepsilon \in[0,1]$, a problem equals to (2). Our first task is to find, for each $\varepsilon \in[0,1]$ and $H_{\varepsilon} \in \Sigma_{\varepsilon}$, a solution $t \mapsto \varphi\left(t, H_{\varepsilon}\right) u_{0}$ of (10). But we will solve this problem in a slightly different way, considering all possible functions in $\left\{B_{\lambda} h_{\varepsilon}^{e}-\tilde{A}_{\lambda}\right\}_{\lambda \in \Gamma, h_{\varepsilon} \in \mathscr{G}_{\mathcal{E}}}$, and therefore, we will denote this space by $\Gamma \diamond \mathscr{G}_{\mathcal{E}}$; that is,

$$
\Gamma \diamond \mathscr{G}_{\varepsilon}=\left\{B_{\lambda} h_{\varepsilon}^{e}-\tilde{A}_{\lambda}\right\}_{\lambda \in \Gamma, h_{\varepsilon} \in \mathscr{G}_{\varepsilon}} .
$$

Remark 10. It is clear that the maps $\mathbb{R} \ni t \mapsto B_{\lambda}(t)$ and $\mathbb{R} \ni t \mapsto \tilde{A}_{\lambda}(t)$ are absolutely continuous, for each $\lambda \in \Gamma$.

\footnotetext{
${ }^{a}$ We once again denote the same.
} 


\section{Non-autonomous dynamical systems and skew-product semiflows for (10)}

In this section we will show that equation (10) generates a non-autonomous dynamical system $\left(\varphi_{\varepsilon}, \theta\right)_{\left(H_{0}^{1}(\Omega), \Sigma_{\varepsilon}\right)}$, for each $\varepsilon \in[0,1]$.

\subsection{Local existence and uniqueness of solutions}

Using Remark 10, Lemma 7 and the results on [24, Chapter 5] we have the following theorem of local existence and uniqueness of solutions.

Theorem 13. Assume that hypotheses (H1) and (C) are satisfied. Then, for each bounded subset $B \subset H_{0}^{1}(\Omega)$, $\varepsilon \in[0,1]$ and $H_{\varepsilon} \in \Gamma \diamond \mathscr{G}_{\varepsilon}$ there exists $\omega=\omega\left(B, \varepsilon, H_{\varepsilon}\right)>0$ such that for each $u_{0} \in B$ there exists a unique function

$$
[0, \omega] \ni t \mapsto \varphi_{\varepsilon}\left(t, H_{\varepsilon}\right) u_{0}
$$

with $\varphi_{\varepsilon}\left(\cdot, H_{\varepsilon}\right) u_{0} \in C^{1}\left([0, \omega], H_{0}^{1}(\Omega)\right)$ satisfying $(10)$; that is, $\varphi_{\varepsilon}\left(0, H_{\varepsilon}\right) u_{0}=u_{0}$ and

$$
\frac{d}{d t} \varphi_{\varepsilon}\left(t, H_{\varepsilon}\right) u_{0}=H_{\varepsilon}\left(t, \varphi_{\varepsilon}\left(t, H_{\varepsilon}\right) u_{0}\right), \text { for } 0<t<\omega .
$$

\subsection{Global existence of solutions}

Assume that (H2) holds true. Following the ideas of [19,26], for any $v \in H_{0}^{1}(\Omega), h_{\varepsilon} \in \mathscr{G}_{\varepsilon}$ and each $\delta>0$ there exists a constant $K_{\delta}>0$ such that

$$
\begin{gathered}
\int_{\Omega} h_{\mathcal{\varepsilon}}^{e}(t, v) v \leqslant \delta\|v\|_{L^{2}(\Omega)}^{2}+K_{\delta}, \\
\int_{\Omega} \Phi_{\varepsilon}(t, v) \leqslant \delta\|v\|_{L^{2}(\Omega)}^{2}+K_{\delta}
\end{gathered}
$$

with $\Phi_{\varepsilon}(t, r)=\int_{0}^{r} h_{\varepsilon}(t, \theta) d \theta$, uniformly in $t \in \mathbb{R}$ and $\varepsilon \in[0,1]$.

Now for each $v \in H_{0}^{1}(\Omega)$, we define the energy functional $L_{b, \varepsilon}(t, v)$ as

$$
L_{b, \varepsilon}(t, v)=\frac{1}{2}\left(\|v\|_{L^{2}(\Omega)}^{2}+b\|v\|_{H_{0}^{1}(\Omega)}^{2}\right)-b \int_{\Omega} \Phi_{\varepsilon}(t, v),
$$

with $b>0$. It is easy to prove that for $\delta \leqslant \frac{1}{2 b}$,

$$
L_{b, \varepsilon}(t, v) \geqslant \frac{b}{2}\|v\|_{H_{0}^{1}(\Omega)}^{2}-b K_{\delta}
$$

and for any $\delta>0$,

$$
L_{b, \varepsilon}(t, v) \leqslant \frac{b \lambda_{1}+2(1+b \delta)}{2 \lambda_{1}}\|v\|_{H_{0}^{1}(\Omega)}^{2}+b K_{\delta},
$$

uniformly in time $t \in \mathbb{R}$, with $\lambda_{1}>0$ the first eigenvalue of $A=-\Delta$ with Dirichlet boundary conditions.

Now, assuming that (H3) holds true and using (12), for a solution $u(t)=\varphi_{\varepsilon}\left(t, H_{\varepsilon}\right) u_{0}$ of (10), where $H_{\varepsilon} \in$ $\Gamma \diamond \mathscr{G}_{\mathcal{E}}$, we have

$$
\begin{aligned}
\frac{d}{d t} L_{b, \varepsilon}(t, u) \leqslant & -\lambda(t)\left(u, u_{t}\right)_{H_{0}^{1}(\Omega)}-\|u\|_{H_{0}^{1}(\Omega)}^{2} \\
& +\left(u, h_{\varepsilon}^{e}(t, u)\right)_{L^{2}(\Omega)}-b\left(\left\|u_{t}\right\|_{L^{2}(\Omega)}^{2}+\lambda(t)\left\|u_{t}\right\|_{H_{0}^{1}(\Omega)}^{2}\right)+C \\
\leqslant & \left(1-\frac{\lambda_{1} \gamma_{1} \eta+2 \delta}{2 \lambda_{1}}\right)\|u\|_{H_{0}^{1}(\Omega)}^{2}+\lambda(t) \frac{1-2 \eta b}{2 \eta}\left\|u_{t}\right\|_{H_{0}^{1}(\Omega)}^{2}+C
\end{aligned}
$$


for $\delta, \eta>0$ and taking $\delta \in\left(\lambda_{1}-\frac{\gamma_{1}}{2}, \lambda_{1}\right), \eta<\frac{2\left(\lambda_{1}-\delta\right)}{\lambda_{1} \gamma_{1}}$ and $b>\frac{1}{2 \eta}$, we have

$$
\frac{d}{d t} L_{b, \varepsilon}(t, u) \leqslant-k L_{b, \varepsilon}(t, u)+C
$$

with $k, C>0$ that do not depend neither on time $t \in \mathbb{R}$ nor on $\varepsilon \in[0,1]$. Therefore,

$$
\|u(t)\|_{H_{0}^{1}(\Omega)}^{2} \leqslant K\left\|u_{0}\right\|_{H_{0}^{1}(\Omega)}^{2} e^{-k t}+C,
$$

for certain constants $k>0$ and $K, C \geqslant 0$ which do not depend on time, $\varepsilon$ and $H_{\varepsilon} \in \Gamma \diamond \mathscr{G}_{\varepsilon}$, and thus we have the global existence of solutions for (10). To summarise, we have so far the following result

Theorem 14. Assume that conditions $(\mathbf{H 1})-(\mathbf{H 3})$ and $(\mathbf{C})$ are satisfied. Then, for each $\varepsilon \in[0,1], H_{\varepsilon} \in \Gamma \diamond \mathscr{G}_{\varepsilon}$ and $u_{0} \in H_{0}^{1}(\Omega)$, equation (10) has a solution $\varphi\left(\cdot, H_{\varepsilon}\right) u_{0}$ defined for all $t \geqslant 0$. Moreover, there exists a bounded subset $B_{0}$ of $H_{0}^{1}(\Omega)$, independent of $\varepsilon \in[0,1]$, such that for each bounded subset $B \subset H_{0}^{1}(\Omega)$, there exists $T=T(B) \geqslant 0$ such that for $t \geqslant T$ we have

$$
\varphi_{\varepsilon}\left(t, H_{\varepsilon}\right) B \subset B_{0} .
$$

Proof. It is simple to see that, using (16), each solution given by Theorem 13 exists for all $t \geqslant 0$. Now define

$$
B_{0}=\left\{v \in H_{0}^{1}(\Omega):\|v\|_{H_{0}^{1}(\Omega)}^{2} \leqslant 2 C\right\},
$$

where $C$ is given in (16). Given $B$ a bounded subset of $H_{0}^{1}(\Omega)$, set $\|B\|=\sup _{v \in B}\|v\|_{H_{0}^{1}(\Omega)}$ and choose

$$
T=\frac{1}{k} \ln \left(\frac{K\|B\|^{2}}{C}\right)
$$

where $k$ and $K$ are given in (16).

Since $\Sigma_{\varepsilon} \subset \Gamma \diamond \mathscr{G}_{\varepsilon}$ and $\Sigma_{\varepsilon}$ is a compact invariant set for the group of translations $\left\{\theta_{t}: t \in \mathbb{R}\right\}$ in $\mathscr{C}_{*}$, a simple consequence of Theorem 14 is the following:

Theorem 15. Assume that conditions $(\mathbf{H 1})-(\mathbf{H 3})$ and $(\mathbf{C})$ are satisfied. Then, for each $\varepsilon \in[0,1]$, equation (10) generates a non-autonomous dynamical system $\left(\varphi_{\varepsilon}, \theta\right)$ in $\left(H_{0}^{1}(\Omega), \Sigma_{\varepsilon}\right)$, where for each $\varepsilon \in[0,1], H_{\varepsilon} \in \Sigma$ and $u_{0} \in H_{0}^{1}(\Omega)$, the function $\mathbb{R}_{+} \ni t \mapsto \varphi_{\varepsilon}\left(t, H_{\varepsilon}\right) u_{0}$ is the unique solution of (10).

\subsection{Skew-product semiflows for (10)}

Using (3), we are able to define, for each $\varepsilon \in[0,1]$, the skew-product semiflow

$$
\left\{\Pi_{\varepsilon}(t): t \geqslant 0\right\} \text { in } \mathbb{X}_{\varepsilon}=H_{0}^{1}(\Omega) \times \Sigma_{\varepsilon}
$$

associated with $\left(\varphi_{\varepsilon}, \theta\right)_{\left(H_{0}^{1}(\Omega), \Sigma_{\varepsilon}\right)}$, by setting

$$
\Pi_{\mathcal{\varepsilon}}(t)\left(u_{0}, H_{\varepsilon}\right)=\left(\varphi_{\varepsilon}\left(t, H_{\varepsilon}\right) u_{0}, \theta_{t} H_{\varepsilon}\right),
$$

for all $t \geqslant 0,\left(u_{0}, H_{\varepsilon}\right) \in \mathbb{X}_{\varepsilon}$ and $\varepsilon \in[0,1]$.

\section{Different attractors for (10)}

In this section we will use the result of Section 2 to obtain different attractors for equation (10), for the different frameworks described in Subsection 1.1. 


\subsection{Global attractors for skew-product semiflows}

In this section, we will use the theory of autonomous equations to find a global attractor for each one of the skew-product semiflows defined by (17).

We first will see a very simple result, that follows from Theorem 14.

Proposition 16. Assume that $(\mathbf{H 1})-(\mathbf{H 3})$ and $(\mathbf{C})$ are satisfied and fix $\varepsilon \in[0,1]$. Then there exists a bounded set $\mathbb{B}_{\varepsilon} \subset \mathbb{X}_{\varepsilon}$ such that given a bounded subset $\mathbb{B} \subset \mathbb{X}_{\varepsilon}$ there exists $T=T(B) \geqslant 0$ such that

$$
\Pi_{\mathcal{\varepsilon}}(t) \mathbb{B} \subset \mathbb{B}_{\varepsilon}, \text { for all } t \geqslant T \text {. }
$$

Proof. Let $B_{0} \subset H_{0}^{1}(\Omega)$ be as in Theorem (14) and define $\mathbb{B}_{\varepsilon} \doteq B_{0} \times \Sigma_{\varepsilon}$. Since $B_{0}$ is bounded in $H_{0}^{1}(\Omega)$ and $\Sigma$ is compact space, we have that $\mathbb{B}_{\varepsilon}$ is bounded in $\mathbb{X}_{\varepsilon}$. Moreover, if $\mathbb{B}$ is a bounded subset of $\mathbb{X}_{\varepsilon}$, we have that $\mathbb{B} \subset B \times \Sigma_{\varepsilon}$, for some bounded set $B \subset H_{0}^{1}(\Omega)$. Hence, if $T$ is as in Theorem 14 we obtain the result.

\subsection{Asymptotical compactness}

In order to obtain a global attractor for each skew-product semiflow, we must prove that the semigroups $\left\{\Pi_{\varepsilon}(t): t \geqslant 0\right\}$ are asymptotically compact. That is our goal for the next few results.

Lemma 17. Define $H_{\lambda}(t, v)=-\tilde{A}_{\lambda}(t) v \in \mathscr{C}_{*}$. Then the problem

$$
\left\{\begin{array}{l}
\dot{u}=H_{\lambda}(t, u), t>0 \\
u(0)=u_{0} \in H_{0}^{1}(\Omega)
\end{array}\right.
$$

has a unique solution $\varphi_{\lambda}(t)$ defined for all $t \geqslant 0$ given by

$$
\varphi_{\lambda}(t) u_{0}=u_{0}-\int_{0}^{t} \tilde{A}_{\lambda}(s) \varphi_{\lambda}(s) u_{0} d s, \text { for all } t \geqslant 0 .
$$

and also, there exists constants $K, k>0$ which do not depend on $\lambda$ such that

$$
\left\|\varphi_{\lambda}(t) u_{0}\right\|_{H_{0}^{1}(\Omega)} \leqslant K\left\|u_{0}\right\|_{H_{0}^{1}(\Omega)} e^{-k t}, \text { for all } t \geqslant 0 .
$$

Proof. The local existence and uniqueness of $\varphi_{*}$ follows from Theorem 13. Proceeding as in Subsection 4.2 with $h_{\varepsilon} \equiv 0$, we can see that we can take $C=0$ in (16) and gives us the global existence of $\varphi_{*}$ and (19). Equation (18) is a simple consequence of the theory of ordinary differential equations, since $-\tilde{A}_{\lambda}(t)$ is a uniformly bounded operator of $H_{0}^{1}(\Omega)$, and the bounds do not depend on $\lambda \in \Gamma$.

Lemma 18. If $H_{\varepsilon}=B_{\lambda} h_{\varepsilon}^{e}-\tilde{A}_{\lambda} \in \Sigma_{\varepsilon}$ and $u_{0} \in H_{0}^{1}(\Omega)$ then

$$
\varphi_{\varepsilon}\left(t, H_{\varepsilon}\right) u_{0}=\varphi_{\lambda}(t) u_{0}+\psi(t)\left(u_{0}, H_{\varepsilon}\right), \text { for each } t \geqslant 0,
$$

where $\psi(t)\left(u_{0}, H_{\varepsilon}\right) \doteq \int_{0}^{t} B_{\lambda}(s) h_{\varepsilon}^{e}\left(s, \varphi_{\varepsilon}\left(s, H_{\varepsilon}\right) u_{0}\right) d s$. Moreover, the map $\psi(t)$ is a compact map from $H_{0}^{1}(\Omega) \times \Sigma_{\varepsilon}$ into $H_{0}^{1}(\Omega)$.

Proof. Clearly the right side of the equation is a solution of (10), thus the uniqueness shows the equality. Now let $B$ be a bounded set of $H_{0}^{1}(\Omega)$. By (16) the set $\mathscr{B} \doteq\left\{\varphi_{\varepsilon}\left(s, H_{\varepsilon}\right) B: s \in[0, t], H_{\varepsilon} \in \Sigma_{\varepsilon}\right\}$ is bounded and thus Lemma 7 ensures that $\cup_{h_{\varepsilon} \in \mathscr{G}_{\varepsilon}} h_{\varepsilon}^{e}([0, t], \mathscr{B})$ is a precompact set of $H^{-1}$. The fact that $B_{\lambda}(t)$ is a uniformly bounded bounded linear operator for $t \in \mathbb{R}$ and $\lambda \in \Gamma$ concludes the proof.

Using these two lemmas, we are able to prove the asymptotical compactness for the skew-product semiflows.

Proposition 19. The skew-product semiflow $\left\{\Pi_{\mathcal{\varepsilon}}(t): t \geqslant 0\right\}$ is asymptotically compact, for each $\varepsilon \in[0,1]$. 
Proof. Let $\left\{u_{n}\right\}_{n \in \mathbb{N}}$ be a bounded sequence in $H_{0}^{1}(\Omega),\left\{H_{\varepsilon, n}\right\}_{n \in \mathbb{N}}$ a bounded sequence in $\Sigma_{\varepsilon}$ and $\left\{t_{n}\right\}_{n \in \mathbb{N}}$ be a real sequence with $t_{n} \rightarrow \infty$ as $n \rightarrow \infty$ and such that the sequence $\left\{\Pi_{\varepsilon}\left(t_{n}\right)\left(u_{n}, H_{\varepsilon, n}\right)\right\}$ is bounded. We have

$$
\Pi_{\varepsilon}\left(t_{n}\right)\left(u_{n}, H_{\varepsilon, n}\right)=\left(\varphi_{\varepsilon}\left(t_{n}, H_{\varepsilon, n}\right) u_{n}, \theta_{t_{n}} H_{\varepsilon, n}\right) \text {, for all } n \in \mathbb{N} \text {. }
$$

Since $\Sigma$ is compact, we can assume, up to a subsequence, that there exists $H_{\varepsilon} \in \Sigma_{\varepsilon}$ such that $H_{\varepsilon}=\mathscr{C}_{*}-$ $\lim _{n \rightarrow \infty} \theta_{t_{n}} H_{\varepsilon, n}$.

Now using Lemma 18 we can write

$$
\varphi_{\varepsilon}\left(t_{n}, H_{\varepsilon, n}\right) u_{n}=\varphi_{*}\left(t_{n}\right) u_{n}+\psi\left(t_{n}\right)\left(u_{n}, H_{\varepsilon, n}\right), \text { for each } n \in \mathbb{N} .
$$

Since $\left\{u_{n}\right\}_{n \in \mathbb{N}}$ is bounded in $H_{0}^{1}(\Omega)$, Lemma 17 implies that $\varphi_{*}\left(t_{n}\right) u_{n} \rightarrow 0$ as $n \rightarrow \infty$. It is now simple to see that the sequence $\left\{\varphi_{\varepsilon}\left(t_{n}, H_{\varepsilon, n}\right) u_{n}\right\}_{n \in \mathbb{N}}$ is precompact in $H_{0}^{1}(\Omega)$, which proves that the sequence $\left\{\Pi_{\varepsilon}\left(t_{n}\right)\left(u_{n}, H_{\varepsilon, n}\right)\right\}$ has a convergent subsequence in $\mathbb{X}_{\varepsilon}$ and concludes the proof.

Now we can join the results of Propositions 16 and 19, together with Theorem 1, to obtain the next theorem.

Theorem 20 (Existence of the global attractor). Assume that $(\mathbf{H 1})-(\mathbf{H 3})$ and $(\mathbf{C})$ hold. Then the skew-product semiflow $\left\{\Pi_{\varepsilon}(t): t \geqslant 0\right\}$ associated with the non-autonomous dynamical system $\left(\varphi_{\varepsilon}, \theta\right)_{\left(H_{0}^{1}(\Omega), \Sigma\right)}$ has a global attractor $\mathbb{A}_{\varepsilon}$ in $\mathbb{X}_{\varepsilon}$ for each $\varepsilon \in[0,1]$. Moreover

$$
\mathbb{A}_{\varepsilon} \subset B_{0} \times \Sigma_{\varepsilon}, \text { for each } \varepsilon \in[0,1],
$$

where $B_{0}$ is the bounded set given in Theorem 14.

We know that the attractors $\mathbb{A}_{\varepsilon}$ can be characterized by

$$
\begin{aligned}
\mathbb{A}_{\varepsilon}=\left\{\left(u_{0}, H_{\varepsilon}\right) \in \mathbb{X}_{\varepsilon}:\right. & \text { there exists a bounded global solution } \eta_{\varepsilon} \\
& \text { of } \left.\left\{\Pi_{\varepsilon}(t): t \geqslant 0\right\} \text { through }\left(u_{0}, H_{\varepsilon}\right)\right\} .
\end{aligned}
$$

It is not difficult to see that we can write $\eta_{\varepsilon}$ as

$$
\eta_{\varepsilon}(t)=\left(\xi_{\varepsilon}(t), \theta_{t} H_{\varepsilon}\right)
$$

where $\xi$ is a global solution of (10); that is, $\varphi_{\varepsilon}\left(t-s, \theta_{s} H_{\varepsilon}\right) \xi_{\varepsilon}(s)=\xi_{\varepsilon}(t)$ for all $t \geqslant s$, and $\xi(0)=u_{0}$. Using Lemma 18 we can write

$$
\xi_{\varepsilon}(t)=\varphi_{\varepsilon}\left(t-s, \theta_{s} H_{\varepsilon}\right) \xi_{\varepsilon}(s)=\varphi_{\lambda}(t-s) \xi_{\varepsilon}(s)+\psi(t-s)\left(\xi_{\varepsilon}(s), \theta_{s} H_{\varepsilon}\right),
$$

where

$$
\begin{aligned}
\psi(t-s)\left(\xi_{\varepsilon}(s), \theta_{s} H_{\varepsilon}\right) & =\int_{0}^{t-s} B_{\theta_{s} \lambda}(r) \theta_{s} h_{\varepsilon}^{e}\left(r, \varphi\left(r, \theta_{s} H_{\varepsilon}\right) \xi_{\varepsilon}(s)\right) d r \\
& =\int_{0}^{t-s} B_{\lambda}(r+s) h_{\varepsilon}^{e}\left(r+s, \xi_{\varepsilon}(r+s)\right) d r \\
& =\int_{s}^{t} B_{\lambda}(r) h_{\varepsilon}^{e}\left(r, \xi_{\varepsilon}(r)\right) d r .
\end{aligned}
$$

Now, making $s \rightarrow-\infty$ in (20), since $\left\{\xi_{\varepsilon}(s)\right\}_{s \in \mathbb{R}}$ is bounded in $H_{0}^{1}(\Omega)$ we obtain, using (19), that

$$
\xi(t)=\int_{-\infty}^{t} B_{\lambda}(r) h_{\varepsilon}^{e}(r, \xi(r)) d r
$$

Using Lemma 7 we can show (following the ideas of [26]) that the solution $\xi_{\varepsilon}$ is bounded in $H^{2}(\Omega) \cap H_{0}^{1}(\Omega)$, and the bound does not depend on the particular $\xi_{\varepsilon}$ (neither it does on $\varepsilon$ ). Hence we obtain that there exists a bounded subset $D_{0}$ of $H^{2}(\Omega) \cap H_{0}^{1}(\Omega)$ such that

$$
\mathbb{A}_{\varepsilon} \subset D_{0} \times \Sigma_{\varepsilon}, \text { for each } \varepsilon \in[0,1] .
$$




\subsection{Other attractors}

Now using Theorem 20 we are able to obtain other attractors for equation (10).

Theorem 21. Assume that (H1)-(H3) and (C) hold true. Then we have, for each $\varepsilon \in[0,1]$, that

(a) the non-autonomous dynamical system $\left(\varphi_{\varepsilon}, \theta\right)_{\left(H_{0}^{1}(\Omega), \Sigma_{\varepsilon}\right)}$ has a uniform attractor $\mathscr{A}_{\varepsilon}$ and

$$
\mathscr{A}_{\varepsilon}=\pi_{H_{0}^{1}(\Omega)} \mathbb{A}_{\varepsilon} \subset D_{0}
$$

(b) the the non-autonomous dynamical system $\left(\varphi_{\varepsilon}, \theta\right)_{\left(H_{0}^{1}(\Omega), \Sigma_{\varepsilon}\right)}$ has a cocycle attractor $\left\{A\left(H_{\varepsilon}\right)\right\}_{H_{\varepsilon} \in \Sigma_{\varepsilon}}$ with $\bigcup_{H_{\varepsilon} \in \Sigma_{\varepsilon}} A\left(H_{\varepsilon}\right) \subset D_{0}$, and

$$
A\left(H_{\varepsilon}\right)=\left\{v \in H_{0}^{1}(\Omega):\left(v, H_{\varepsilon}\right) \in \mathbb{A}_{\varepsilon}\right\}
$$

(c) for each $H_{\varepsilon} \in \Sigma_{\varepsilon}$, the evolution process $\left\{T_{H_{\varepsilon}}(t, s): t \geqslant s\right\}$ given by

$$
T_{H_{\varepsilon}}(t, s)=\varphi_{\varepsilon}\left(t-s, \theta_{s} H_{\varepsilon}\right), \text { for all } t \geqslant s,
$$

has a pullback attractor $\left\{A_{H_{\varepsilon}}(t)\right\}_{t \in \mathbb{R}}$ with $\bigcup_{t \in \mathbb{R}} A_{H_{\varepsilon}}(t) \subset D_{0}$, and

$$
\mathbb{A}_{\varepsilon}=\bigcup_{H_{\varepsilon} \in \Sigma_{\varepsilon}}\left[\bigcup_{t \in \mathbb{R}} A_{H_{\varepsilon}}(t) \times\left\{H_{\varepsilon}\right\}\right] .
$$

where $D_{0}$ is given in (21).

Proof. Using Theorem 20, we have easily that item (a) follows from Theorem 5, item (b) from Theorem 3 and item (c) from Theorem 4.

\section{Upper semicontinuity of attractors}

This section is devoted to study the upper semicontinuity of the semigroups $\left\{\Pi_{\mathcal{E}}(t): t \geqslant 0\right\}$ as perturbations of $\left\{\Pi_{0}(t): t \geqslant 0\right\}$, as a part of the study described in Subsection 1.2. So far, we have treated the family of equations (6) (and equivalently, (10)) individually for each $\varepsilon \in[0,1]$, but now it is time to look at all these equations together at once.

Assuming that (H1)-(H3) and (C) hold, we obtained so far a family of semigroups $\left\{\Pi_{\varepsilon}(t): t \geqslant 0\right\}$ in $\mathbb{X}_{\varepsilon}=$ $H_{0}^{1}(\Omega) \times \Sigma_{\varepsilon}$, and for each $\varepsilon$, a global attractor $\mathbb{A}_{\varepsilon}$.

Definition 11. We say that a family $\left\{K_{\varepsilon}\right\}_{\varepsilon \in[0,1]}$ is upper semicontinuous at 0 in a metric space $(X, d)$ if given sequences $\left\{\varepsilon_{n}\right\}_{n \in \mathbb{N}} \subset(0,1]$ and $x_{n} \in K_{\varepsilon_{n}}$, with $\varepsilon_{n} \rightarrow 0^{+}$as $n \rightarrow \infty$, there exists a convergent subsequence of $\left\{x_{n}\right\}_{n \in \mathbb{N}}$ with limit belonging to the closure of $K_{0}$ in $(X, d)$.

The previous definition is equivalent to the following: a family $\left\{K_{\varepsilon}\right\}_{\varepsilon \in[0,1]}$ is upper semicontinuous at 0 in a metric space $(X, d)$ if

$$
\lim _{\varepsilon \rightarrow 0^{+}} \mathrm{d}_{H}\left(K_{\mathcal{\varepsilon}}, K_{0}\right)=0 .
$$

To prove the upper semicontinuity of $\left\{\mathbb{A}_{\varepsilon}\right\}_{\varepsilon \in[0,1]}$, we set the base space as $H_{0}^{1}(\Omega) \times \mathscr{C}_{*}$, with a metric $\mathfrak{d}$ defined by

$$
\mathfrak{d}\left[\left(u_{1}, H_{1}\right),\left(u_{2}, H_{2}\right)\right]=\left\|u_{1}-u_{2}\right\|_{H_{0}^{1}(\Omega)}+d_{*}\left(H_{1}, H_{2}\right),
$$

for all $\left(u_{1}, H_{1}\right),\left(u_{2}, H_{2}\right) \in H_{0}^{1}(\Omega) \times \mathscr{C}_{*}$.

Before studying the upper semicontinuity of the family of attractors $\left\{\mathbb{A}_{\varepsilon}\right\}_{\varepsilon \in[0,1]}$, we will need some convergence results. 
Lemma 22. If (H4) holds and $h_{\varepsilon} \in \mathscr{G}_{\varepsilon}$, we have

$$
\sup _{t \in \mathbb{R}}\left|h_{\varepsilon}(t, s)-f(s)\right| \leqslant \beta(\varepsilon)\left(1+|s|^{\rho-1}\right),
$$

for all $s \in \mathbb{R}$.

Proof. Let $h_{\varepsilon} \in \mathscr{G}_{\varepsilon}$ and $\left\{t_{m}\right\}$ be a real sequence such that

$$
\lim _{m \rightarrow \infty} d_{2}\left(h_{\mathcal{\varepsilon}}, \theta_{t_{m}} g_{\varepsilon}\right)=\lim _{m \rightarrow \infty} \sup _{t, s \in \mathbb{R}}\left|h_{\mathcal{\varepsilon}}(t, s)-\theta_{t_{m}} g_{\varepsilon}(t, s)\right|=0,
$$

where $d_{2}$ is as in Subsection 3.1. Hence

$$
\begin{gathered}
\sup _{t \in \mathbb{R}}\left|h_{\mathcal{\varepsilon}}(t, s)-f(s)\right| \leqslant \sup _{t \in \mathbb{R}}\left|h_{\mathcal{\varepsilon}}(t, s)-g_{\mathcal{\varepsilon}}\left(t+t_{m}, s\right)\right|+\sup _{t \in \mathbb{R}}\left|g_{\varepsilon}\left(t+t_{m}, s\right)-f(s)\right| \\
\leqslant d_{2}\left(h_{\mathcal{\varepsilon}}, \theta_{t_{m}} g_{\varepsilon}\right)+\sup _{t \in \mathbb{R}}\left|g_{\varepsilon}(t, s)-f(s)\right| \\
\leqslant d_{2}\left(h_{\mathcal{\varepsilon}}, \theta_{t_{m}} g_{\varepsilon}\right)+\beta(\varepsilon)\left(1+|s|^{\rho-1}\right),
\end{gathered}
$$

and making $m \rightarrow \infty$ we obtain the result.

Lemma 23. Assume that $(\mathbf{H 4})$ and $(\mathbf{C})$ hold. Then there exists a constant $c>0$ such that

$$
\sup _{h_{\varepsilon} \in \mathscr{G}_{\varepsilon}} \sup _{\varepsilon \in \mathbb{R}}\left\|h_{\varepsilon}^{e}(t, u)-f^{e}(u)\right\|_{L^{\frac{2 n}{n+2}(\Omega)}} \leqslant c \beta(\varepsilon)\left(1+\|u\|_{H_{0}^{1}(\Omega)}^{\rho-1}\right)
$$

for all $u \in H_{0}^{1}(\Omega)$.

Proof. Proceeding as in Lemma 7 and using Lemma 22 we have

$$
\begin{aligned}
\sup _{t \in \mathbb{R}} \| h_{\varepsilon}^{e}(t, u) & -f^{e}(u) \|_{L^{\frac{2 n}{n+2}(\Omega)}}=\left[\int_{\Omega}\left|h_{\varepsilon}^{e}(t, u)-f^{e}(u)\right|^{\frac{2 n}{n+2}}\right]^{\frac{n+2}{2 n}} \\
& \leqslant \beta(\varepsilon)\left[\int_{\Omega}\left(1+|u|^{\rho-1}\right)^{\frac{2 n}{n+2}}\right]^{\frac{n+2}{2 n}} \leqslant \tilde{c} \beta(\varepsilon)\left(1+\|u\|_{L^{\frac{n(\rho-1)}{2}}(\Omega)}^{\rho-1}\right) \\
& \leqslant c \beta(\varepsilon)\left(1+\|u\|_{H_{0}^{1}(\Omega)}^{\rho-1}\right),
\end{aligned}
$$

and the result follows.

Corollary 24. If (H4) and (C) hold, we have that there exists a constant $\hat{C}>0$ such that

$$
\sup _{h_{\varepsilon} \in \mathscr{G}_{\varepsilon}} d_{2}^{e}\left(h_{\varepsilon}^{e}, f^{e}\right) \leqslant \hat{C} \beta(\varepsilon)
$$

Proposition 25. Assume that $(\mathbf{H 4})$ and $(\mathbf{C})$ hold true and consider the family $\left\{\Sigma_{\varepsilon}\right\}_{\varepsilon \in[0,1]}$ given in Definition 10 . Then we have that given sequences $\left\{\varepsilon_{n}\right\}_{n \in \mathbb{N}} \subset(0,1]$ with $\varepsilon_{n} \rightarrow 0^{+}$and $H_{n} \in \Sigma_{\varepsilon_{n}}$, for each $n \in \mathbb{N}$, there exists a convergent subsequence of $\left\{H_{n}\right\}_{n \in \mathbb{N}}$ in $\left(\mathscr{C}_{*}, d_{*}\right)$, with its limit belonging to $\Sigma_{0}$.

Proof. Since $H_{n} \in \Sigma_{\varepsilon_{n}}$, item (b) of Lemma 12 implies that there exists $\lambda_{n} \in \Gamma$ and $h_{n} \in \mathscr{G}_{\varepsilon_{n}}$ such that

$$
H_{n}=B_{\lambda_{n}} h_{n}^{e}-\tilde{A}_{\lambda_{n}}, \text { for each } n \in \mathbb{N} .
$$

Since $\Gamma$ is compact (recall Remark 7), there exists a subsequence $\left\{\lambda_{n_{k}}\right\}$ that converges to a function $\lambda_{0}$ in $\left(\mathscr{C}_{1}, d_{1}\right)$. Now, Corollary 24 shows that $d_{2}^{e}\left(h_{n}^{e}, f^{e}\right) \leqslant \hat{C} \beta\left(\varepsilon_{n}\right) \rightarrow 0$ as $n \rightarrow \infty$ and hence $h_{n}^{e}$ converges to $f^{e}$ in $\left(\mathscr{C}_{2}^{e}, d_{2}^{e}\right)$. Therefore, we can easily see that $H_{n_{k}}$ converges to $B_{\lambda_{0}} f^{e}-\tilde{A}_{\lambda_{0}}$ in $\left(\mathscr{C}_{*}, d_{*}\right)$, which is in $\Sigma_{0}$ by item (a) of Lemma 12.

With these preliminaries results, we are able to begin the proof of the upper semicontinuity of the family of the global attractors $\left\{\mathbb{A}_{\varepsilon}\right\}_{\varepsilon \in[0,1]}$ of the skew-product semiflows, at $\varepsilon=0$. 
Lemma 26. If $\left\{\left(u_{\mathcal{\varepsilon}}, H_{\varepsilon}\right)\right\}_{\varepsilon \in(0,1]}$ is such that $\left(u_{\mathcal{\varepsilon}}, H_{\varepsilon}\right) \subset \mathbb{X}_{\varepsilon}$ and there exists $\left(u_{0}, H_{0}\right) \in \mathbb{X}_{0}$ such that $\mathfrak{d}\left[\left(u_{\varepsilon}, H_{\varepsilon}\right),\left(u_{0}, H_{0}\right)\right] \rightarrow$ 0 as $\varepsilon \rightarrow 0^{+}$, we have

$$
\mathfrak{d}\left[\Pi_{\varepsilon}(t)\left(u_{\varepsilon}, H_{\varepsilon}\right), \Pi_{0}(t)\left(u_{0}, H_{0}\right)\right] \stackrel{\varepsilon \rightarrow 0^{+}}{\longrightarrow} 0, \text { for each } t \geqslant 0 .
$$

Proof. We can write

$$
\Pi(t)\left(u_{\varepsilon}, H_{\varepsilon}\right)=\left(\varphi_{\varepsilon}\left(t, H_{\varepsilon}\right) u_{\varepsilon}, \theta_{t} H_{\varepsilon}\right), \text { for each } \varepsilon \in[0,1] .
$$

Since $d_{*}\left(H_{\varepsilon}, H_{0}\right) \rightarrow 0$ and $\left\{\theta_{t}: t \geqslant 0\right\}$ is continuous in $\mathscr{C}_{*}$ for each $t \geqslant 0$, we easily obtain that $d_{*}\left(\theta_{t} H_{\varepsilon}, \theta_{t} H_{0}\right) \rightarrow$ 0 , as $\varepsilon \rightarrow 0^{+}$.

It remains to show that $\left\|\varphi_{\varepsilon}\left(t, H_{\varepsilon}\right) u_{\varepsilon}-\varphi_{0}\left(t, H_{0}\right) u_{0}\right\|_{H_{0}^{1}(\Omega)} \rightarrow 0$, as $\varepsilon \rightarrow 0^{+}$. Using Lemma 18 , we can write

$$
\begin{aligned}
& \varphi_{\varepsilon}\left(t, H_{\varepsilon}\right) u_{\varepsilon}-\varphi_{0}\left(t, H_{0}\right) u_{0} \\
& \quad=\varphi_{\lambda_{\varepsilon}}(t) u_{\varepsilon}-\varphi_{\lambda_{0}}(t) u_{0}+\int_{0}^{t}\left[B_{\lambda_{\varepsilon}}(s) h_{\varepsilon}^{e}\left(s, \varphi_{\varepsilon}\left(s, H_{\varepsilon}\right) u_{\varepsilon}\right)-B_{\lambda_{0}}(s) f^{e}\left(\varphi_{0}\left(s, H_{0}\right) u_{0}\right)\right] d s,
\end{aligned}
$$

where we assumed $H_{\varepsilon}=B_{\lambda_{\varepsilon}} h_{\varepsilon}^{e}-\tilde{A}_{\lambda_{\varepsilon}}$ and $H_{0}=B_{\lambda_{0}} f^{e}-\tilde{A}_{\lambda_{0}}$.

We have, using Lemma 17, we obtain

$$
\begin{gathered}
\left\|\varphi_{\lambda_{\varepsilon}}(t) u_{\varepsilon}-\varphi_{\lambda_{0}}(t) u_{0}\right\|_{H_{0}^{1}(\Omega)} \leqslant\left\|\varphi_{\lambda_{\varepsilon}}(t) u_{\varepsilon}-\varphi_{\lambda_{\varepsilon}}(t) u_{0}\right\|_{H_{0}^{1}(\Omega)}+\left\|\varphi_{\lambda_{\varepsilon}}(t) u_{0}-\varphi_{\lambda_{0}}(t) u_{0}\right\|_{H_{0}^{1}(\Omega)} \\
\leqslant K\left\|u_{\varepsilon}-u_{0}\right\|_{H_{0}^{1}(\Omega)} e^{-k t}+\left\|\varphi_{\lambda_{\varepsilon}}(t) u_{0}-\varphi_{\lambda_{0}}(t) u_{0}\right\|_{H_{0}^{1}(\Omega)} .
\end{gathered}
$$

Again, using Lemma 17, item 2 of Remark 7 and the Gronwall inequality, we obtain that

$$
\left\|\varphi_{\lambda_{\varepsilon}}(t) u_{\varepsilon}-\varphi_{\lambda_{0}}(t) u_{0}\right\|_{H_{0}^{1}(\Omega)}=O(\varepsilon) .
$$

For the second term, we have

$$
\begin{aligned}
\| B_{\lambda_{\varepsilon}}(s) h_{\varepsilon}^{e}\left(s, \varphi_{\varepsilon}\left(s, H_{\varepsilon}\right) u_{\varepsilon}\right) & -B_{\lambda_{0}}(s) f^{e}\left(\varphi_{0}\left(s, H_{0}\right) u_{0}\right) \|_{H_{0}^{1}(\Omega)} \\
& \leqslant\left\|B_{\lambda_{\varepsilon}}(s)\left[h_{\varepsilon}^{e}\left(s, \varphi_{\varepsilon}\left(s, H_{\varepsilon}\right) u_{\varepsilon}\right)-f^{e}\left(\varphi_{\varepsilon}\left(s, H_{\varepsilon}\right) u_{\varepsilon}\right)\right]\right\|_{H_{0}^{1}(\Omega)} \\
& +\left\|\left[B_{\lambda_{\varepsilon}}(s)-B_{\lambda_{0}}(s)\right] f^{e}\left(\varphi_{0}\left(s, H_{0}\right) u_{0}\right)\right\|_{H_{0}^{1}(\Omega)}
\end{aligned}
$$

and hence, using again item 2 of Remark 7 we obtain that

$$
\begin{aligned}
\int_{0}^{t} \| B_{\lambda_{\varepsilon}}(s) h_{\varepsilon}^{e}\left(s, \varphi_{\varepsilon}\left(s, H_{\varepsilon}\right) u_{\varepsilon}\right) & -B_{\lambda_{0}}(s) f^{e}\left(\varphi_{0}\left(s, H_{0}\right) u_{0}\right) \|_{H_{0}^{1}(\Omega)} \\
& \leqslant O(\varepsilon)+\int_{0}^{t}\left\|\varphi_{\varepsilon}\left(s, H_{\varepsilon}\right) u_{\varepsilon}-\varphi_{0}\left(s, H_{0}\right) u_{0}\right\|_{H_{0}^{1}(\Omega)} d s .
\end{aligned}
$$

Finally, joining the estimates and applying again the Gronwall inequality, we obtain that

$$
\left\|\varphi_{\varepsilon}\left(t, H_{\varepsilon}\right) u_{\varepsilon}-\varphi_{0}\left(t, H_{0}\right) u_{0}\right\|_{H_{0}^{1}(\Omega)} \leqslant O(\varepsilon),
$$

and concludes the result.

We can prove the following:

Lemma 27. If $\left\{\left(u_{\varepsilon}, H_{\varepsilon}\right)\right\}_{\varepsilon \in(0,1]}$ is such that $\left(u_{\varepsilon}, H_{\varepsilon}\right) \in \mathbb{A}_{\varepsilon}$ and

$$
\lim _{\varepsilon \rightarrow 0^{+}} \mathfrak{d}\left[\left(u_{\varepsilon}, H_{\varepsilon}\right),\left(u_{0}, H_{0}\right)\right]=0
$$

for some $\left(u_{0}, H_{0}\right) \in H_{0}^{1}(\Omega) \times \mathscr{C}_{*}$, then $\left(u_{0}, H_{0}\right) \in \mathbb{A}_{0}$. 
Proof. Using the characterization of global attractors in Subsection 2.1, we know that through each $\left(u_{\varepsilon}, H_{\varepsilon}\right) \in \mathbb{A}_{\varepsilon}$ we have a global bounded solution $\xi_{\varepsilon}: \mathbb{R} \rightarrow \mathbb{X}_{\varepsilon}$ of $\left\{\Pi_{\varepsilon}(t): t \geqslant 0\right\}$. To show that $\left(u_{0}, H_{0}\right) \in \mathbb{A}_{0}$, it is sufficient to prove that through $\left(u_{0}, H_{0}\right)$ there exists a global bounded solution $\xi_{0}: \mathbb{R} \rightarrow \mathbb{X}_{0}$ of $\left\{\Pi_{0}(t): t \geqslant 0\right\}$.

For any $t \geqslant 0$, we define $\xi_{0}(t)=\Pi(t)\left(u_{0}, H_{0}\right)$. Since $\left\{\Pi_{0}(t): t \geqslant 0\right\}$ has a global attractor, the set $\xi_{0}([0, \infty))$ is bounded.

We now use an induction argument to define the solution for negative values of $t$. Consider the family $\left\{\xi_{\varepsilon}(-1)\right\}_{\varepsilon \in[0,1]}$, which we can write as

$$
\xi_{\varepsilon}(-1)=\left(u_{\varepsilon}(-1), \theta_{-1} H_{\varepsilon}\right) .
$$

Using (21), we have that the family $\left\{u_{\mathcal{\varepsilon}}(-1)\right\}_{\varepsilon \in[0,1]}$ and hence there exists a sequence $\varepsilon_{1, n} \rightarrow 0^{+}$and a point $u_{-1} \in H_{0}^{1}(\Omega)$ such that

$$
u_{\varepsilon_{1, n}}(-1) \rightarrow u_{-1}, \text { in } H_{0}^{1}(\Omega)
$$

We define then $\xi_{0}(-1)=\left(u_{-1}, \theta_{-1} H_{0}\right)$ and $\xi(t)=\Pi_{0}(t+1)\left(u_{-1}, \theta_{-1} H_{0}\right)$, for $-1 \leqslant t<0$. Clearly, using Lemma 26, we have

$$
\left(u_{\varepsilon_{1, n}}, H_{\varepsilon_{1, n}}\right)=\Pi_{\varepsilon_{1, n}}(1) \xi_{\varepsilon_{1, n}}(-1) \rightarrow \Pi_{0}(1) \xi_{0}(-1),
$$

and thus $\left(u_{0}, H_{0}\right)=\xi_{0}(0)=\Pi_{0}(1) \xi_{0}(-1)$.

Proceeding inductively, for each $k \in \mathbb{N}$, we obtain a subsequence $\left\{\varepsilon_{k, n}\right\}_{n \in \mathbb{N}}$ of $\left\{\varepsilon_{k-1, n}\right\}_{n \in \mathbb{N}}$ with $\varepsilon_{k, n} \rightarrow 0^{+}$as $n \rightarrow \infty$ and a point $u_{-k} \in H_{0}^{1}(\Omega)$ such that if $\xi_{\varepsilon}(-k)=\left(u_{\varepsilon}(-k), \theta_{-k} H_{\varepsilon}\right)$ we have

$$
u_{\varepsilon_{k, n}}(-j) \rightarrow u_{-j}, \text { for all } j=1, \cdots, k .
$$

Defining $\xi_{0}(-k)=\left(u_{-k}, \theta_{-k} H_{0}\right)$ and $\xi_{0}(t)=\Pi_{0}(t+k) \xi_{0}(-k)$ for $-k \leqslant t<-k+1$, we have that

$$
\left(u_{\varepsilon_{k, n}}, H_{\varepsilon_{k, n}}\right)=\Pi_{\varepsilon_{k, n}}(1) \xi_{\varepsilon_{k, n}}(-j) \rightarrow \Pi_{0}(-j+1) \xi_{0}(-j+1), \text { for each } j=1 \cdots, k,
$$

and thus $\left(u_{0}, H_{0}\right)=\xi_{0}(0)=\Pi_{0}(k) \xi_{0}(-k)$.

Therefore, we obtain that $\xi_{0}: \mathbb{R} \rightarrow \mathbb{X}_{0}$ is a bounded global solution of $\left\{\Pi_{0}(t): t \geqslant 0\right\}$ through $\left(u_{0}, H_{0}\right)$, which implies that $\left(u_{0}, H_{0}\right) \in \mathbb{A}_{0}$ and concludes the proof.

Now, we can easily prove the upper semicontinuity of the family of global attractors $\left\{\mathbb{A}_{\varepsilon}\right\}_{\varepsilon \in[0,1]}$.

Theorem 28. The family of global attractors $\left\{\mathbb{A}_{\varepsilon}\right\}_{\varepsilon \in[0,1]}$ is upper semicontinuous at 0 .

Proof. If $\left\{\varepsilon_{n}\right\}_{n \in \mathbb{N}} \subset(0,1]$, with $\varepsilon_{n} \rightarrow 0$ and $\left(u_{n}, H_{n}\right) \in \mathbb{A}_{\varepsilon_{n}}$, it is clear that there exists a convergent subsequence of $\left\{\left(u_{n}, H_{n}\right)\right\}_{n \in \mathbb{N}}$ to a point $\left(u_{0}, H_{0}\right) \in \mathbb{X}_{0}$ (using (21)). Hence, Lemma 27 shows that $\left(u_{0}, H_{0}\right) \in \mathbb{A}_{0}$, which concludes the proof.

\subsection{Upper semicontinuity for other attractors}

As in immediate consequence of Theorem 28 we have (recall Theorem 21):

Corollary 29. Assume that $(\mathbf{H 1})-(\mathbf{H 4})$ and $(\mathbf{C})$ hold true. Then we have that

- the family of uniform attractors $\left\{\mathscr{A}_{\mathcal{E}}\right\}_{\varepsilon \in[0,1]}$;

- the family of cocycle attractors $\left\{A\left(H_{\varepsilon}\right)\right\}_{H_{\varepsilon} \in \Sigma_{\varepsilon}}$ and

- the family of pullback attractors $\left\{A_{H_{\varepsilon}}(t)\right\}_{t \in \mathbb{R}}$

are upper semicontinuous at 0 in $H_{0}^{1}(\Omega)$. 


\section{Topological structure of attractors}

Following the results of [7, Section 3], we will study the structure of the global attractors $\mathbb{A}_{\varepsilon}$ for the skewproduct semiflows $\left\{\Pi_{\varepsilon}(t): t \geqslant 0\right\}$ and using this structure we obtain informations about the structure for the other attractors defined in Theorem 21.

\subsection{Structure of $\mathbb{A}_{0}$}

To study the structure of the global attractors $\mathbb{A}_{\varepsilon}$, we will study in more detail the structure of the global attractor $\mathbb{A}_{0}$ and we will make the following assumption:

There exists a finite number of isolated equilibia $\mathscr{E}=\left\{e_{1}, \cdots, e_{p}\right\}$ of

$$
\left\{\begin{array}{cc}
-\Delta u=f(u), & \text { in } \Omega \\
u=0, & \text { on } \partial \Omega .
\end{array}\right.
$$

With this assumption, we define

$$
\mathbb{E}_{i}=\left\{e_{i}\right\} \times \Sigma_{0} \subset \mathbb{X}_{0}, \text { for } i=1, \cdots, p .
$$

Lemma 30. Each set $\mathbb{E}_{i}, i=1, \cdots, p$, is invariant by the skew-product semiflow $\left\{\Pi_{0}(t): t \geqslant 0\right\}$ and $\mathbb{E}_{i} \subset \mathbb{A}_{0}$, for each $i=1, \cdots, p$.

Proof. Clearly, if we take $H_{0} \in \Sigma_{0}$, the solution $[0, \infty) \ni t \mapsto \varphi_{0}\left(t, H_{0}\right) e_{i}$ of (10) is the constant solution $\varphi_{0}\left(t, H_{0}\right) e_{i}=e_{i}$, for all $t \geqslant 0$; hence

$$
\Pi_{0}(t)\left(e_{i}, H_{0}\right)=\left(e_{i}, \theta_{t} H_{0}\right) \in \mathbb{E}_{i}, \text { for all } t \geqslant 0 .
$$

Conversely, if $t \geqslant 0$ and $H \in \Sigma_{0}$ are given, set $H_{0}=\theta_{-t} H$. We have

$$
\Pi_{0}(t)\left(e_{i}, H\right)=\left(e_{i}, \theta_{t} H\right)=\left(e_{i}, H_{0}\right),
$$

therefore $\left(e_{i}, H_{0}\right) \in \Pi_{0}(t) \mathbb{E}_{i}$. The last claim follows since $\mathbb{E}_{i}$ is a bounded invariant subset of $\mathbb{X}_{0}$.

We can now define a functional on $\mathbb{X}_{0}$, which will help us understand the intern structure of $\mathbb{A}_{0}$.

Definition 12. Define the functional $V: \mathbb{X}_{0} \rightarrow \mathbb{R}$ by

$$
V\left(v, H_{0}\right)=\frac{1}{2}\|v\|_{H_{0}^{1}(\Omega)}^{2}-\int_{\Omega} W(v),
$$

where $W(r)=\int_{0}^{r} f(\theta) d \theta$, for each $\left(v, H_{0}\right) \in \mathbb{X}_{0}$.

Lemma 31. Let $\left\{\Pi_{0}(t): t \geqslant 0\right\}$ be the skew-product semiflow defined in (17) for $\varepsilon=0$. If $\left(u_{0}, H_{0}\right) \in \mathbb{X}_{0}$ and $V$ is the functional in defined in (23), we have that

(a) the map $[0, \infty) \ni t \mapsto V\left(\Pi_{0}(t)\left(u_{0}, H_{0}\right)\right)$ is non-increasing, for each $\left(u_{0}, H_{0}\right) \in \mathbb{X}_{0}$ and it is constant in $\mathbb{E}_{i}$, $i=1, \cdots, p$.

(b) If the map $[0, \infty) \ni t \mapsto V\left(\Pi_{0}(t)\left(u_{0}, H_{0}\right)\right)$ is constant, then $\left(u_{0}, H_{0}\right) \in \mathbb{E}_{i}$, for some $i=1, \cdots, p$.

Proof. Since $V\left(\Pi_{0}(t)\left(u_{0}, H_{0}\right)\right)=V\left(\varphi_{0}\left(t, H_{0}\right) u_{0}, \theta_{t} H_{0}\right)$, it is a straightforward computation to see, if $H_{0}=B_{\lambda} f^{e}-$ $\tilde{A}_{\lambda}$ for some $\lambda \in \Gamma$, that

$$
\frac{d}{d t} V\left(\Pi_{0}(t)\left(u_{0}, H_{0}\right)\right)=-\left\|\frac{d}{d t} \varphi\left(t, H_{0}\right) u_{0}\right\|_{L^{2}(\Omega)}^{2}-\lambda(t)\left\|\frac{d}{d t} \varphi\left(t, H_{0}\right) u_{0}\right\|_{H_{0}^{1}(\Omega)}^{2} \leqslant 0,
$$


since $0<\gamma_{0} \leqslant \lambda(t)$, for all $t \in \mathbb{R}$; so the map $[0, \infty) \ni t \mapsto V\left(\Pi_{0}(t)\left(u_{0}, H_{0}\right)\right)$ is non-increasing, and it is clearly constant in each $\mathbb{E}_{i}$.

Now, if map $[0, \infty) \ni t \mapsto V\left(\Pi_{0}(t)\left(u_{0}, H_{0}\right)\right)$ is constant, we have that $\varphi_{0}\left(t, H_{0}\right) u_{0}=u_{0}$, for all $t \geqslant 0$, and hence $u_{0}$ is a equilibrium of $-\Delta u=f(u)$ in $H_{0}^{1}(\Omega)$, which implies that $u_{0}=e_{i}$, for some $i=1, \cdots, p$.

Definition 13. Let $\left(u_{0}, H_{0}\right) \in \mathbb{A}_{0}$. We define the $\omega$-limit of $\left(u_{0}, H_{0}\right)$ by

$$
\begin{array}{r}
\omega\left(u_{0}, H_{0}\right)=\left\{(u, H) \in \mathbb{A}_{0}: \text { there exists a sequence } t_{n} \rightarrow \infty\right. \\
\text { such that } \left.\Pi_{0}\left(t_{n}\right)\left(u_{0}, H_{0}\right) \stackrel{n \rightarrow \infty}{\longrightarrow}(u, H)\right\},
\end{array}
$$

and if $\xi: \mathbb{R} \rightarrow \mathbb{A}_{0}$ is a global solution of $\left\{\Pi_{0}(t): \geqslant 0\right\}$ through $\left(u_{0}, H_{0}\right)$, we define the $\alpha_{\xi}$-limit of $\left(u_{0}, H_{0}\right)$ by

$$
\begin{aligned}
\alpha_{\xi}\left(u_{0}, H_{0}\right)=\{(u, H) \in & \mathbb{A}_{0}: \text { there exists a sequence } t_{n} \rightarrow \infty \\
& \text { such that } \left.\xi\left(-t_{n}\right) \stackrel{n \rightarrow \infty}{\longrightarrow}(u, H)\right\},
\end{aligned}
$$

It is a well known result, since $\left\{\Pi_{0}(t): t \geqslant 0\right\}$ has a global attractor $\mathbb{A}_{0}$, that both $\omega\left(u_{0}, H_{0}\right)$ and $\alpha_{\xi}\left(u_{0}, H_{0}\right)$ are non-empty, compact, invariant for $\left\{\Pi_{0}(t): t \geqslant 0\right\}$ and connected. With these, we can prove the following result.

Lemma 32. For any $\left(u_{0}, H_{0}\right) \in \mathbb{A}_{0}$ and any global solution $\xi$ through $\left(u_{0}, H_{0}\right)$, there exists $i, j=1, \cdots, p$ such that

$$
\omega\left(u_{0}, H_{0}\right) \subset \mathbb{E}_{i} \quad \text { and } \quad \alpha_{\xi}\left(u_{0}, H_{0}\right) \subset \mathbb{E}_{j} .
$$

Moreover, if $i=j$, then $u_{0}=e_{i}$.

Proof. Let $(u, H) \in \omega\left(u_{0}, H_{0}\right)$ and $t_{n} \rightarrow \infty$ such that $\Pi_{0}\left(t_{n}\right)\left(u_{0}, H_{0}\right) \rightarrow(u, H)$. Since $V$ is a continuous functional in $\mathbb{X}_{0}$, we have that $V\left(\Pi_{0}\left(t_{n}\right)\left(u_{0}, H_{0}\right)\right) \rightarrow V(u, H)$, as $n \rightarrow \infty$.

Since $V\left(\Pi_{0}(\cdot)\left(u_{0}, H_{0}\right)\right)$ is non-increasing and has a convergent subsequence, we obtain that

$$
V\left(\Pi_{0}(t)\left(u_{0}, H_{0}\right)\right) \rightarrow V(u, H), \text { as } t \rightarrow \infty .
$$

Hence, if $\left(u_{1}, H_{1}\right)$ is any point in $\omega\left(u_{0}, H_{0}\right)$, we have that $V\left(u_{1}, H_{1}\right)=V(u, H)$. Since $\omega\left(u_{0}, H_{0}\right)$ is invariant for $\left\{\Pi_{0}(t): t \geqslant 0\right\}$ we have that $V\left(\Pi_{0}(t)(u, H)\right)=V(u, H)$, and then $[0, \infty) \ni t \mapsto V\left(\Pi_{0}(t)(u, H)\right)$ is constant, which implies that $(u, H) \in \mathbb{E}_{i}$, for some $i=1, \cdots, p$. The connectedness of $\omega\left(u_{0}, H_{0}\right)$ shows us that $\omega\left(u_{0}, H_{0}\right) \subset$ $\mathbb{E}_{i}$.

The proof for $\alpha_{\xi}\left(u_{0}, H_{0}\right)$ is analogous and the last assertion is straightforward

Proposition 33. The family $\mathfrak{E}=\left\{\mathbb{E}_{1}, \cdots, \mathbb{E}_{p}\right\}$ is a disjoint family of isolated invariants for $\left\{\Pi_{0}(t): t \geqslant 0\right\}$; that is, $\mathbb{E}_{i} \subset \mathbb{A}_{0}, \Pi_{0}(t) \mathbb{E}_{i}=\mathbb{E}_{i}$ for all $t \geqslant 0$, there exists $\delta>0$ such that $\mathbb{E}_{i}$ is the maximal invariant set for $\left\{\Pi_{0}(t): t \geqslant 0\right\}$ in

$$
\mathscr{O}_{\delta}\left(\mathbb{E}_{i}\right)=\left\{(v, H) \in \mathbb{X}_{0}:\left\|v-e_{i}\right\|_{H_{0}^{1}(\Omega)}<\delta\right\},
$$

for each $i=1, \cdots, p$, and also $\mathbb{E}_{i} \cap \mathbb{E}_{j}=\varnothing$, if $1 \leqslant i \neq j \leqslant p$.

Proof. It only remains to prove that there exists $\delta>0$ such that $\mathbb{E}_{i}$ is the maximal invariant set in $\mathscr{O}_{\delta}\left(\mathbb{E}_{i}\right)$. Let $\delta=\frac{1}{2} \min _{1 \leqslant i \neq j \leqslant p}\left\|e_{i}-e_{j}\right\|_{H_{0}^{1}(\Omega)}$.

If $\mathbb{E}_{i}$ is not the maximal invariant in $\mathscr{O}_{\delta}\left(\mathbb{E}_{i}\right)$, there exists a global solution $\xi$ of $\left\{\Pi_{0}(t): t \geqslant 0\right\}$ such that $\xi(\mathbb{R}) \subset \mathscr{O}_{\delta}\left(\mathbb{E}_{i}\right)$, with $\xi(\mathbb{R}) \backslash \mathbb{E}_{i} \neq \varnothing$. But then the previous lemma shows that $\omega(\xi(0)) \subset \mathbb{E}_{i}$ and $\alpha_{\xi}(\xi(0)) \subset \mathbb{E}_{i}$, which implies that $\xi(0)=\left(e_{i}, H_{0}\right)$, and therefore $\xi(\mathbb{R}) \subset \mathbb{E}_{i}$, so we reached a contradiction.

All these results combined show us that the semigroup $\left\{\Pi_{0}(t): t \geqslant 0\right\}$ is, in fact, a generalized gradient semigroup (see $[7,15,26]$ for more details) with disjoint family of isolated invariants $\mathfrak{E}=\left\{\mathbb{E}_{1}, \cdots, \mathbb{E}_{p}\right\}$, and as a consequence, we can write the global attractor $\mathbb{A}_{0}$ as

$$
\mathbb{A}_{0}=\bigcup_{i=1}^{p} \mathbb{W}^{u}\left(\mathbb{E}_{i}\right),
$$


where

$$
\begin{aligned}
\mathbb{W}^{u}\left(\mathbb{E}_{i}\right)=\{(u, H) & \in \mathbb{A}_{0}: \text { there exists a global solution } \xi \text { of }\left\{\Pi_{0}(t): t \geqslant 0\right\} \\
& \text { with } \left.\xi(0)=(u, H) \text { such that } \mathfrak{d}\left(\xi(t), \mathbb{E}_{i}\right) \rightarrow 0, \text { as } t \rightarrow-\infty\right\} .
\end{aligned}
$$

If $H_{0} \in \Sigma_{0}$, there exists $\lambda \in \Gamma$ such that $H_{0}=B_{\lambda} f^{e}-\tilde{A}_{\lambda}$. Thus we can define

$$
\begin{aligned}
& W^{u}\left(e_{i}, H_{0}\right)=\left\{u \in H_{0}^{1}(\Omega): \text { there exists a global solution } \eta \text { of }(10) \text { for } H_{0}\right. \\
& \text { with } \left.\eta(0)=u \text { such that }\left\|\eta(t)-e_{i}\right\|_{H_{0}^{1}(\Omega)} \rightarrow 0, \text { as } t \rightarrow-\infty\right\} .
\end{aligned}
$$

It is simple to see that

$$
\mathbb{W}^{u}\left(\mathbb{E}_{i}\right)=W^{u}\left(e_{i}, H_{0}\right) \times\left\{H_{0}\right\}, \text { for each } i=1, \cdots, p,
$$

and thus we have that

$$
\mathbb{A}_{0}=\bigcup_{i=1}^{p} \bigcup_{H_{0} \in \Sigma_{0}} W^{u}\left(e_{i}, H_{0}\right) \times\left\{H_{0}\right\},
$$

and Theorem 21 shows us that the uniform attractor $\mathscr{A}_{0}$ of the non-autonomous dynamical system $\left(\varphi_{0}, \theta\right)_{\left(H_{0}^{1}(\Omega), \Sigma_{0}\right)}$ is given by

$$
\mathscr{A}_{0}=\bigcup_{i=1}^{p} \bigcup_{H_{0} \in \Sigma_{0}} W^{u}\left(e_{i}, H_{0}\right) .
$$

Moreover, the cocycle attractor $\left\{A\left(H_{0}\right)\right\}_{H_{0} \in \Sigma_{0}}$ of the non-autonomous dynamical system $\left(\varphi_{0}, \theta\right)_{\left(H_{0}^{1}(\Omega), \Sigma_{0}\right)}$ is given by

$$
A\left(H_{0}\right)=\bigcup_{i=1}^{p} W^{u}\left(e_{i}, H_{0}\right) \text {, for each } H_{0} \in \Sigma_{0} .
$$

And finally, for each $H_{0} \in \Sigma_{0}$, the pullback attractor $\left\{A_{H_{0}}(t)\right\}_{t \in \mathbb{R}}$ of the evolution process $T_{H_{0}}(t, s)=\varphi_{0}(t-$ $\left.s, \theta_{s} H_{0}\right)$ is given by

$$
A_{H_{0}}(t)=\bigcup_{i=1}^{p} W^{u}\left(e_{i}, \theta_{t} H_{0}\right) \text {, for all } t \in \mathbb{R} \text { and each } H_{0} \in \Sigma_{0} .
$$

\section{Remark 11.}

1. If $H_{0}=B_{\gamma} f^{e}-\tilde{A}_{\gamma}$, defining

$$
\mathscr{A}(t)=A_{H_{0}}(t), \text { for each } t \in \mathbb{R},
$$

we obtain the pullback attractor for (5); that is, for each $t \in \mathbb{R}$, we have

$$
\mathscr{A}(t)=\{\eta(t): \eta \text { is a bounded global solution of (5) }\} \text {. }
$$

2. If $\gamma(\cdot)$ is a constant function, equation (5) is autonomous, $\Sigma_{0}=\left\{B_{\gamma} f^{e}-\tilde{A}_{\gamma}\right\}$ is a singleton and we obtain, as a particular case, that the autonomous system generated by (5) is a gradient semigroup with a finite collection of equilibria $\mathscr{E}=\left\{e_{1}, \cdots, e_{p}\right\}$ and that $\mathbb{A}_{0}=\mathscr{A}_{0} \times \Sigma_{0}$, where $\mathscr{A}_{0}=W^{u}\left(e_{i}, H_{0}\right)$ and $H_{0}=B_{\gamma} f^{e}-$ $\tilde{A} \gamma$. 


\subsection{Structure of $\mathbb{A}_{\varepsilon}$}

In this subsection we prove that, under suitable conditions, the attractors $\mathbb{A}_{\varepsilon}$ inherit the same generalized gradient structure from $\mathbb{A}_{0}$. To this end, we first need to take the following fact in account: we have, following the ideas of the proof of Lemma 26 , if $H_{\varepsilon} \rightarrow H_{0}$ in $\left(\mathscr{C}_{*}, d_{*}\right)$, that

$$
\lim _{\varepsilon \rightarrow 0^{+}} \mathfrak{d}\left[\Pi_{\varepsilon}(t)\left(u, H_{\varepsilon}\right), \Pi_{0}(t)\left(u, H_{0}\right)\right]=0,
$$

uniformly for $t$ in bounded subsets of $\mathbb{R}, u$ in bounded subsets of $H_{0}^{1}(\Omega)$.

With all these considerations and previous results, we are able to state the following structural result.

Theorem 34. Assume that hypotheses (H1)-(H4), (C) and (F) hold true. Assume also that

(a) for each $\varepsilon \in(0,1]$ there exists a disjoint family of isolated invariants $\mathfrak{E}_{\varepsilon}=\left\{\mathbb{E}_{1, \varepsilon}, \cdots, \mathbb{E}_{p, \varepsilon}\right\}$ for $\left\{\Pi_{\varepsilon}(t): t \geqslant\right.$ $0\}$ such that

$$
\mathfrak{d}_{H}\left[\mathbb{E}_{i, \varepsilon}, \mathbb{E}_{i}\right]+\mathfrak{d}_{H}\left[\mathbb{E}_{i}, \mathbb{E}_{i, \varepsilon}\right] \rightarrow 0 \text {, as } \varepsilon \rightarrow 0^{+} ;
$$

(b) there exists $\varepsilon_{0}>0$ and neighborhoods $\mathbb{V}_{i}$ of $\mathbb{E}_{i}$ such that $\mathbb{E}_{i, \varepsilon}$ is the maximal invariant set of $\left\{\Pi_{\mathcal{\varepsilon}}(t): t \geqslant 0\right\}$ in $\mathbb{V}_{i}$, for each $i=1, \cdots, p$ and $0<\varepsilon \leqslant \varepsilon_{0}$.

Then there exists $\varepsilon_{1}>0$ such that $\left\{\Pi_{\varepsilon}(t): t \geqslant 0\right\}$ is a generalized gradient semigroup with a disjoint family of isolated invariants $\mathfrak{E}_{\varepsilon}$, for each $0 \leqslant \varepsilon \leqslant \varepsilon_{1}$. Moreover, for $0 \leqslant \varepsilon \leqslant \varepsilon_{1}$, we have

$$
\mathbb{A}_{\varepsilon}=\bigcup_{i=1}^{p} \mathbb{W}^{u}\left(\mathbb{E}_{i, \varepsilon}\right) .
$$

Proof. The proof of this theorem is analogous to the proof of [15, Theorem 1.5], with the aid of Proposition 25.

\subsection{Global hyperbolic solutions for (10)}

Let $\xi_{\varepsilon}$ be a global solution of $\left\{\Pi_{\varepsilon}(t): t \geqslant 0\right\}$ in $\mathbb{A}_{\varepsilon}$. Thus we have that there exists $H_{\varepsilon} \in \Sigma_{\varepsilon}$ such that $\xi_{\varepsilon}(t)=\left(\eta_{\varepsilon}(t), \theta_{t} H_{\varepsilon}\right)$, for all $t \in \mathbb{R}$, where $\eta_{\varepsilon}$ is a global bounded solution of (10).

Writing $H_{\varepsilon}=B_{\lambda} h_{\varepsilon}^{e}-\tilde{A}_{\lambda}$, for some $\lambda \in \Gamma$ and $h_{\varepsilon} \in \mathscr{C}_{2}$, we can consider the variational problem for the solution $\eta_{\varepsilon}$, given by

$$
\left\{\begin{array}{l}
u_{t}-\lambda(t) \Delta u_{t}-\Delta u=D_{s} h_{\varepsilon}^{e}\left(t, \eta_{\varepsilon}(t)\right) u, \text { in } \Omega \\
u=0, \text { on } \partial \Omega,
\end{array}\right.
$$

where $\left[D_{s} h_{\varepsilon}^{e}\left(t, \eta_{\varepsilon}(t)\right) u\right](x)=\partial_{s} h_{\varepsilon}\left(t, \eta_{\varepsilon}(t)(x)\right) u(x)$, a.e. $x \in \Omega$, for $u \in H_{0}^{1}(\Omega)$.

This equation generates a linear evolution process $\left\{L_{\xi_{\varepsilon}}(t, s): t \geqslant s\right\} \subset \mathscr{L}\left(H_{0}^{1}(\Omega)\right)$, where $u(t)=L_{\xi_{\varepsilon}}(t, s) u_{0}$ is the solution at time $t$ of (24) with $u(s)=u_{0}$.

Definition 14. We say that $\left\{L_{\xi_{\varepsilon}}(t, s): t \geqslant s\right\}$ has an exponential dichotomy if there exists a family of projections $\{Q(t)\}_{t \in \mathbb{R}}$ in $\mathscr{L}\left(H_{0}^{1}(\Omega)\right)$ satisfying:

(a) $Q(t) L_{\xi_{\varepsilon}}(t, s)=L_{\xi_{\varepsilon}}(t, s) Q(s)$, for all $t \geqslant s$.

(b) The restriction $\left.L_{\xi_{\varepsilon}}(t, s)\right|_{R(Q(s))}, t \geqslant s$, is an isomorphism from $R(Q(s))$ into $R(Q(t))$; and we denote its inverse by $L_{\xi_{\varepsilon}}(s, t): R(Q(t)) \rightarrow R(Q(s))$.

(c) There are constants $\omega>0$ and $M \geqslant 1$ such that

$$
\begin{aligned}
& \left\|L_{\xi_{\varepsilon}}(t, s)(I-Q(s))\right\|_{\mathscr{L}\left(H_{0}^{1}(\Omega)\right)} \leqslant M e^{-\omega(t-s)}, \text { for } t \geqslant s ; \\
& \left\|L_{\xi_{\varepsilon}}(s, t) Q(t)\right\|_{\mathscr{L}\left(H_{0}^{1}(\Omega)\right)} \leqslant M e^{\omega(s-t)}, \text { for } s<t .
\end{aligned}
$$


In this case, we say that $\xi_{\varepsilon}$ is a hyperbolic global solution of $\left\{\Pi_{\varepsilon}(t): t \geqslant 0\right\}$.

Now we make the following assumption:

$$
\begin{aligned}
& \text { Each global solution } \xi_{i, H_{0}}^{*}(t)=\left(e_{i}, \theta_{t} H_{0}\right), t \in \mathbb{R} \text { and } \\
& \qquad H_{0} \in \Sigma_{0} \text {, is hyperbolic. }
\end{aligned}
$$

Remark 12. Since $\Sigma_{0}=\left\{B_{\lambda} f^{e}-\tilde{A}_{\lambda}\right\}_{\lambda \in \Gamma}$, we have a family $\left\{\xi_{i, \lambda}^{*}\right\}_{\lambda \in \Gamma}$ of hyperbolic global solutions of $\left\{\Pi_{0}(t): t \geqslant\right.$ $0\}$, where $\xi_{i, \lambda}^{*}=\xi_{i, H_{0}}^{*}$ for $H_{0}=B_{\lambda} f^{e}-\tilde{A}_{\lambda}$.

Now, proceeding as in [16, Section 7.2] and [26], we have the following result:

Proposition 35. Assume that hypotheses $(\mathbf{H 1})-(\mathbf{H 5}),(\mathbf{C}),(\mathbf{F})$ and $(\mathbf{H y})$ hold true. Thus, there exist $\varepsilon_{0}>0$ and $\delta>0$ such that for each $i=1, \cdots, p, \lambda \in \Gamma$ and $\varepsilon \in\left(0, \varepsilon_{0}\right]$, there exist a unique $h_{\varepsilon}^{*} \in \mathscr{G}_{\varepsilon}$ and a unique bounded global solution $\eta_{i, \lambda, \varepsilon}^{*}: \mathbb{R} \rightarrow \mathscr{A}_{\varepsilon}$ of (10), with $H_{\varepsilon}=B_{\lambda} h_{\varepsilon}^{*, e}-\tilde{A}_{\lambda}$, which we denote by $H_{\varepsilon}^{\lambda}$, such that:

(i) the function $\mathbb{R} \ni t \mapsto \xi_{i, \lambda, \varepsilon}^{*}(t)=\left(\eta_{i, \lambda, \varepsilon}^{*}(t), \theta_{t} H_{\varepsilon}^{\lambda}\right) \in \mathbb{A}_{\varepsilon}$ is a hyperbolic global solution of $\left\{\Pi_{\mathcal{\varepsilon}}(t): t \geqslant 0\right\}$;

(ii) $\sup _{t \in \mathbb{R}} \mathfrak{d}\left[\xi_{i, \lambda, \varepsilon}^{*}(t),\left(e_{i}, \theta_{t} H_{0}^{\lambda}\right)\right]<\delta$; where $H_{0}^{\lambda}=B_{\lambda} f^{e}-\tilde{A}_{\lambda}$, and

(iii) $\sup _{t \in \mathbb{R}} \mathfrak{d}\left[\xi_{i, \lambda, \varepsilon}^{*}(t),\left(e_{i}, \theta_{t} H_{0}^{\lambda}\right)\right] \rightarrow 0$, as $\varepsilon \rightarrow 0^{+}$.

Moreover, if $\xi_{\mathcal{\varepsilon}}$ is a global solution of $\left\{\Pi_{\mathcal{\varepsilon}}(t): t \geqslant 0\right\}$ such that $\mathfrak{d}\left[\xi_{\mathcal{\varepsilon}}(t), \xi_{i, \lambda, \varepsilon}^{*}(t)\right]<\delta$ for all $t \geqslant 0(t \leqslant 0)$ then

$$
\mathfrak{d}\left[\xi_{\varepsilon}(t), \xi_{i, \lambda, \varepsilon}^{*}(t)\right] \rightarrow 0 \text { as } t \rightarrow \infty(t \rightarrow-\infty)
$$

With this proposition, we can construct a family $\mathfrak{E}_{\varepsilon}=\left\{\mathbb{E}_{1, \varepsilon}, \cdots, \mathbb{E}_{p, \varepsilon}\right\}$, satifying the conditions of Theorem 34. In fact, for each $\lambda \in \Gamma$, let

$$
\mathbb{D}_{i, \varepsilon}(\lambda)=\bigcup_{t \in \mathbb{R}} \xi_{i, \lambda, \varepsilon}^{*}(t)=\bigcup_{t \in \mathbb{R}}\left(\eta_{i, \lambda, \varepsilon}^{*}(t), \theta_{t} H_{\varepsilon}^{\lambda}\right), \text { for each } i=1, \cdots, p,
$$

and define

$$
\mathbb{E}_{i, \varepsilon}=\bigcup_{\lambda \in \Gamma} \mathbb{D}_{i, \varepsilon}(\lambda), \text { for each } i=1, \cdots, p .
$$

Now, as an immediate consequence of Theorem 34 and Proposition 35, we have:

Theorem 36. Assume that hypotheses (H1)-(H5), (C), (F) and (Hy) hold true. Consider the disjoint family of isolated invariants $\mathfrak{E}_{\varepsilon}=\left\{\mathbb{E}_{1, \varepsilon}, \cdots, \mathbb{E}_{p, \varepsilon}\right\}$ for $\left\{\Pi_{\mathcal{\varepsilon}}(t): t \geqslant 0\right\}$ given by (25).

Then there exists $\varepsilon_{1}>0$ such that $\left\{\Pi_{\mathcal{E}}(t): t \geqslant 0\right\}$ is a generalized gradient semigroup with a disjoint family of isolated invariants $\mathfrak{E}_{\mathcal{E}}$, for each $0 \leqslant \varepsilon \leqslant \varepsilon_{1}$. Moreover, for $0 \leqslant \varepsilon \leqslant \varepsilon_{1}$, we have

$$
\mathbb{A}_{\varepsilon}=\bigcup_{i=1}^{p} \mathbb{W}^{u}\left(\mathbb{E}_{i, \varepsilon}\right),
$$

and

$$
\mathbb{W}^{u}\left(\mathbb{E}_{i, \varepsilon}\right)=\bigcup_{\lambda \in \Gamma} \mathbb{W}^{u}\left(\mathbb{D}_{i, \varepsilon}(\lambda)\right) .
$$

With this result, we can derive structures for the other types of attractors, as we did for $\mathbb{A}_{0}$; namely, we have that the uniform attractor of $\left(\varphi_{\varepsilon}, \theta\right)_{\left(H_{0}^{1}(\Omega), \Sigma_{\varepsilon}\right)}$ is given by

$$
\mathscr{A}_{\varepsilon}=\pi_{H_{0}^{1}(\Omega)} \mathbb{A}_{\varepsilon}=\bigcup_{i=1}^{p} W^{u}\left(\mathscr{E}_{i, \varepsilon}\right),
$$


where $\mathscr{E}_{i, \varepsilon}=\bigcup_{\lambda \in \Gamma} \bigcup_{t \in \mathbb{R}} \eta_{i, \lambda, \varepsilon}^{*}(t)$. Also, the cocycle attractor for $\left(\varphi_{\varepsilon}, \theta\right)_{\left(H_{0}^{1}(\Omega), \Sigma_{\varepsilon}\right)}$ is given by

$$
A\left(H_{\varepsilon}\right)=\bigcup_{i=1}^{p} W^{u}\left(\mathscr{E}_{i, \varepsilon}(\lambda)\right)
$$

where $\mathscr{E}_{i, \varepsilon}(\lambda)=\bigcup_{t \in \mathbb{R}} \eta_{i, \lambda, \varepsilon}^{*}(t)$ and $H_{\varepsilon}=B_{\lambda} h_{\varepsilon}^{e}-\tilde{A}_{\lambda}$.

Lastly, if $H_{\varepsilon} \in \Sigma_{\varepsilon}$, the pullback attractor $\left\{A_{H_{\varepsilon}}(t)\right\}_{t \in \mathbb{R}}$ of the evolution process $T_{H_{\varepsilon}}(t, s)=\varphi_{\varepsilon}\left(t-s, \theta_{s} H_{\varepsilon}\right)$ is given by

$$
A_{H_{\varepsilon}}(t)=\bigcup_{i=1}^{p} W^{u}\left(\eta_{i, \lambda, \varepsilon}^{*}\right)(t), \text { for all } t \in \mathbb{R}
$$

where

$$
\begin{aligned}
& W^{u}\left(\xi_{i, \lambda, \varepsilon}^{*}\right)(t)=\left\{u \in H_{0}^{1}(\Omega) \text { : there exists a global solution } \eta_{\varepsilon} \text { of }(10) \text { for } H_{\varepsilon}\right. \\
& \text { with } \left.\eta_{\varepsilon}(t)=u \text { such that }\left\|\eta_{\varepsilon}(s)-\eta_{i, \lambda, \varepsilon}^{*}(s)\right\|_{H_{0}^{1}(\Omega)} \rightarrow 0, \text { as } s \rightarrow-\infty\right\} .
\end{aligned}
$$

\subsection{Further remarks}

As discussed extensively in [5] and [7], we could do the analysis presented in this work, removing condition (C) by assuming only that we have a semigroup of translations $\left\{\theta_{t}: t \geqslant 0\right\}$ in $\mathscr{G}_{\varepsilon}$ with a global attractor $\Xi_{\varepsilon} \subset \mathscr{G}_{\varepsilon}$. Thus, despite some complications with notations, the results would remain unchanged, replacing $\mathscr{G}_{\varepsilon}$ by $\Xi_{\varepsilon}$. This, in turn, allows us to give a more precise description of the attractors when $g_{\varepsilon}$ is, for instance, asymptotically autonomous; since in this case, we notice that $\Xi_{\varepsilon}$ is a singleton, which is significantly smaller than $\mathscr{G}_{\varepsilon}$.

\section{References}

[1] E.C. Aifantis, (1980), On the problem of diffusion in solids, Acta Mechanica, 37, No 3, 265-296. doi 10.1007/BF01202949

[2] K. Kuttler and E.C. Aifantis, (1987), Existence and uniqueness in nonclassical diffusion, Quarterly of Applied Mathematics, 45, No 3, 549-560.

[3] K. Kuttler and E.C. Aifantis, (1988), Quasilinear Evolution Equations in Nonclassical Diffusion, SIAM Journal on Mathematical Analysis, 19, No 1, 110-120. doi 10.1137/0519008

[4] C.T. Anh and T.Q. Bao, (2010), Pullback attractors for a class of non-autonomous nonclassical diffusion equations, Nonlinear Analysis: Theory, Methods \& Applications, 73, No 2, 399-412. doi 10.1016/j.na.2010.03.031

[5] M.C. Bortolan, T. Caraballo, A.N. Carvalho and J.A. Langa, (2013), Skew product semiflows and Morse decomposition, Journal of Differential Equations, 255, No 8, 2436-2462. doi 10.1016/j.jde.2013.06.023

[6] M.C. Bortolan and A.N. Carvalho, (2015), Strongly damped wave equation and its Yosida approximations, Topological Methods in Nonlinear Analysis, 46, No 2, 563-602. doi 10.12775/TMNA.2015.059

[7] M.C. Bortolan, A.N. Carvalho and J.A. Langa, (2014), Structure of attractors for skew product semiflows, Journal of Differential Equations, 257, No 2, 490-522. doi 10.1016/j.jde.2014.04.008

[8] R. Camassa and D. D. Holm, (1993), An integrable shallow water equation with peaked solitons, Physical Review Letters 71, 1661-1665. doi 10.1103/PhysRevLett.71.1661

[9] T. Caraballo, A.N. Carvalho, J.A. Langa and F. Rivero, (2010), Existence of pullback attractors for pullback asymptotically compact processes, Nonlinear Analysis: Theory, Methods \& Applications, 72, No 3-4, 1967-1976. doi 10.1016/j.na.2009.09.037

[10] T. Caraballo, A.N. Carvalho, J.A. Langa and F. Rivero., (2011), A non-autonomous strongly damped wave equation: Existence and continuity of the pullback attractor, Nonlinear Analysis: Theory, Methods \& Applications, 74, No 6, 2272-2283. doi 10.1016/j.na.2010.11.032

[11] T. Caraballo, G. Łukaszewicz and J. Real, (2006), Pullback attractors for asymptotically compact nonautonomous dynamical systems, Nonlinear Analysis: Theory, Methods \& Applications, 64, No 3, 484-498. doi 10.1016/j.na.2005.03.111

[12] T. Caraballo and A.M. Márquez-Durán, (2013), Existence, uniqueness and asymptotic behavior of solutions for a nonclassical diffusion equation with delay, Dynamics of Partial Differential Equations, 10, No 3, 267-281. doi 10.4310/dpde.2013.v10.n3.a3 
[13] T. Caraballo, A.M. Márquez-Durán and F. Rivero, (2015), Well-Posedness and Asymptotic Behavior of a Nonclassical Nonautonomous Diffusion Equation with Delay, International Journal of Bifurcation and Chaos, 25, No 14, 1540021, 11 pages. doi 10.1142/S0218127415400210

[14] T. Caraballo, J.C. Jara, J.A. Langa and Z. Liu, (2013), Morse Decomposition of Attractors for Non-autonomous Dynamical Systems, Advanced Nonlinear Studies 13, No 2, 309-329. doi 10.1515/ans-2013-0204

[15] A. N. Carvalho and J.A. Langa, (2009), An extension of the concept of gradient semigroups which is stable under perturbation, Journal of Differential Equations, 246, No 7, 2646-2668. doi 10.1016/j.jde.2009.01.007

[16] A. N. Carvalho, J.A. Langa and J.C. Robinson, (2013), Attractors for infinite-dimensional non-autonomous dynamical systems, Springer, New York. doi 10.1007/978-1-4614-4581-4

[17] V.V. Chepyzhov and M.I. Vishik, (2002), Attractors for Equations of Mathematical Physics, Colloquium Publications, American Mathematical Society, Providence, RI.

[18] O. Ladyzhenskaya, (1991), Attractors for Semigroups and Evolution Equations, Cambridge University Press, Cambridge.

[19] J. K. Hale, (1989), Asymptotic Behavior of Dissipative System, American Mathematical Society, Providence, RI.

[20] J. K. Hale, (1980), Ordinary Differential Equations, Robert E. Kriege Publishing Company, Huntington, NY.

[21] Z. Hu and Y. Wang, (2012), Pullback attractors for a nonautonomous nonclassical diffusion equation with variable delay, Journal of Mathematical Physics, 53, No 7, 17 pages. doi 10.1063/1.4736847

[22] P. E. Kloeden and M. Rasmussen, (2011), Nonautonomous Dynamical Systems, Mathematical Surveys and Monographs, American Mathematical Society, Providence, RI. doi 10.1090/surv/176

[23] Q.-Z. Ma, Y.-F. Liu and F.-H. Zhang, (2012), Global attractors in $H^{1}\left(\mathbb{R}^{N}\right)$ for Nonclassical Diffusion Equations, Discrete Dynamics in Nature and Society, Article ID 672762, 16 pages. doi 10.1155/2012/672762

[24] A. Pazy, (1983), Semigroups of Linear Operators and Applications to Partial Differential Equations, Springer-Verlag, New York. doi 10.1007/978-1-4612-5561-1

[25] P.J. Chen and M.E. Gurtin, (1968), On a theory of heat conduction involving two temperatures, Journal of Applied Mathematics and Physics (ZAMP), 19, No 4, 614-627. doi 10.1007/BF01594969

[26] F. Rivero, (2013), Time dependent perturbation in a non-autonomous non-classical parabolic equation, Discrete and Continuous Dynamical Systems - Series B, 18, No 1, 209-221. doi 10.3934/dcdsb.2013.18.209

[27] G. R. Sell, (1967), Nonautonomous Differential Equations and Topological Dynamics I. The Basic Theory, Transactions of the American Mathematical Society, 127, No 2, 241-262. doi 10.2307/1994645

[28] G. R. Sell and Y. You, (2002), Dynamics of Evolutionary Equations, Springer-Verlag, New York. doi 10.1007/978-14757-5037-9

[29] C.Y. Sun, S.Y. Wang and C.K. Zhong, (2007), Global Attractors for a Nonclassical Diffusion Equation, Acta Mathematica Sinica, English Series, 23, No 7, 1271-1280. doi 10.1007/s10114-005-0909-6

[30] C. Sun and M. Yang, (2008), Dynamics of the nonclassical diffusion equations , Asymptotic Analysis, 59, No 1-2, 51-81. doi 10.3233/ASY-2008-0886

[31] S. Wang, D. Li and C. Zhong, (2006), On the dynamics of a class of nonclassical parabolic equations, Journal of Mathematical Analysis and Applications 317, No 2, 565-582. doi 10.1016/j.jmaa.2005.06.094 
OUP4 Sciences. All rights reserved. 